\title{
Cleonisio Leite Rodrigues
}

\section{Espectro do comprometimento cognitivo na neurocisticercose: \\ diferenças de acordo com a fase da doença}

Tese apresentada à Faculdade de Medicina da Universidade de São Paulo para obtenção de título de Doutor em Ciências

Programa de Neurologia

Orientador: Prof. Dr. José Antônio Livramento

São Paulo 
Dados Internacionais de Catalogação na Publicação (CIP)

Preparada pela Biblioteca da

Faculdade de Medicina da Universidade de São Paulo

Creprodução autorizada pelo autor

\section{Rodrigues, Cleonisio Leite}

Espectro do comprometimento cognitivo na neurocisticercose : diferenças de acordo com a fase da doença / Cleonisio Leite Rodrigues.-- São Paulo, 2011.

Tese(doutorado)--Faculdade de Medicina da Universidade de São Paulo.

Programa de Neurologia.

Orientador: José Antônio Livramento.

Descritores: 1.Neurocisticercose 2.Cognição 3.Demência 4.Imagem por ressonância magnética

USP/FM/DBD-221/11 


\section{DEDICATÓRIA}


Dedico esta tese a meus pais, pelo exemplo de luta, união, abnegação e inquestionável apoio nos momentos cruciais de minha trajetória A meus irmãos, minha namorada e o restante da minha família, pelo companheirismo, compreensão e apoio. Agradeço a Deus, por vocês estarem presentes na minha vida. 
AGRADECIMENTOS 
Aos pacientes que, em busca de alívio de seus anseios e dores, acreditaram neste projeto e possibilitaram que suas patologias fossem melhor estudadas e compreendidas. Aos voluntários, pela disposição para contribuir com o projeto.

Ao Dr. Daniel Ciampi de Andrade, amigo, companheiro fiel de infinitas horas de trabalho na residência, pós-graduação e em todas as etapas deste projeto. Amizade sedimentada à base de confiança, metas comuns, respeito e admiração mútuos. Grande nome da nova geração de neurologistas e de pesquisadores na área de neurologia funcional e dor.

Ao Prof. Dr. José Antônio do Livramento, por me permitir ser seu orientando, pela exatidão na forma de trabalhar e ensinar, desde a época de meu estágio no laboratório Spina França. Orientador ágil, pontual e de uma objetividade incomum.

Ao Prof. Dr. Paulo Caramelli, o co-orientador desta tese, porém idealizador e orientador de todo projeto da linha de pesquisa desde seu nascimento. Alicerce científico fundamental de todos os momentos desta empreitada. Profissional exemplar, um dos grandes nomes da neurologia cognitiva brasileira, mestre na arte de incentivar e de apoiar. Para mim e tantos outros que estão iniciando suas carreiras, um grande exemplo.

Ao Prof. Dr. Luís dos Ramos Machado, pelas ideias sempre pertinentes, pelo apoio incondicional durante todo o projeto, além do auxílio na melhor compreensão de nossos resultados. Neurologista clínico e referência nacional em neuroinfecção, ensinou-me as bases do estudo de líquor e abriu as portas de seu ambulatório, para que pudéssemos aprofundar um terreno mal compreendido da patologia para qual dedicou boa parte de seu tempo nas últimas décadas. 
Ao Prof. Dr. Ronaldo Abraham, pela amizade, por seu senso de organização invejável e por sua atuação na base de nosso projeto como o recrutamento de pacientes e acolhida em Taubaté. Exemplo prévio de realização de uma tese premiada na área de neurocisticercose.

Ao Prof. Dr. Ricardo Nitrini, como coordenador da pós-graduação da Neurologia, professor da cadeira de estudos científicos em neurologia e membro da banca de qualificação, encorajou-me e realizou comentários frutíferos a respeito deste projeto, sempre que teve oportunidade.

Ao Prof. Dr. Paulo Marchiori, médico de conhecimento e sabedoria incomuns, pelo apoio, ensinamentos e orientação durante toda a residência e estágio de complementação especializada.

Ao Prof. Dr. Milberto Scaff, pelo apoio para que este estudo fosse realizado com êxito no departamento, que teve a sua chefia por vários anos.

Ao Prof. Dr. Manoel Jacobsen Teixeira, pelo apoio direto e indireto e que permitiu que este projeto tivesse pleno êxito.

Aos Professores Dra. Cláudia Leite, Dr. Leandro Lucato e a todos médicos da radiologia da FMUSP pela contribuição em parte significativa deste estudo no que concerne à classificação radiológica e estadiamento dos doentes

Ao Dr. Luiz Henrique, pelos ensinamentos na residência e pelo apoio na elaboração do projeto da linha de pesquisa e coleta de dados, máxime quando foram avaliados alguns pacientes do ambulatório de epilepsia.

À Dra. Cláudia Porto, pelos ensinamentos na preparação e utilização do questionário de avaliação cognitiva e funcional.

A Lucas Massaroppe, pelo inestimável contribuição na realização da trabalhosa análise estatística do projeto.

A todos os residentes do triênio 2004, 2005 e 2006, pelo apoio e companhia nas árduas porém insubstituíveis horas da residência, em 
especial, ao Dr. Caio Simioni, amigo e incentivador e aos demais, Dra. Ana Paula, Dr. Norberto e Dr. Lécio.

Aos demais médicos colaboradores do ambulatório de neuroinfecção: Dr. Hélio e Dra. Ana.

A todos os médicos assistentes da neurologia do Hospital das Clínicas da FMUSP, em especial, Dra. Teresa, Dra. Angelina, Dr. Comerlatti, Dr. Brotto, etc.

Aos funcionários do departamento neurologia: Maria de Lourdes, Márcia Benetti, Vanessa Ramos, Sipriana Santos, Sueli Martins, Reiko Uchizono, Vera Lúcia, Elizabete Silva, Vera Maria de Paula, Daniela Cristina, Sandra da Silva e as demais. Apoio logístico fundamental.

À Comissão de Aperfeiçoamento de Pessoal de Ensino Superior (CAPES), pela bolsa recebida em parte do período de pós-graduação.

Aos meus amigos, que me acompanharam nesses anos, Raphael, Rodrigo, Diógenes, Léo, Alexandre e Marcela, Tiago e Renata, Celso e Karine. Márcio, Carlos Márcio, André e Vivi, Hilton, Renato, Breno, Flávio e Sílvia, André e Kenya, Paulo Roberto, Ernesto e Bárbara, dentre outros, pelo incentivo.

Agradecimento especial ao Prof. Dr. Armênio Aguiar dos Santos, professor de Fisiologia Humana da Universidade Federal do Ceará, pelo meu estágio de pesquisa de 1997 a 2001 no laboratório escola prof. Luiz Capelo, onde foi gerado o embrião de minha vocação para pesquisa científica e tive a oportunidade de publicar vários artigos em revistas internacionais de estudos experimentais. Este agradecimento estende-se a todos os contemporâneos da época, em especial ao Dr. Francisco de Assis Aquino Gondim e ao prof. Dr. Francisco Hélio Rola. 


\section{Epígrafe}

"Todas as coisas devem ser feitas da forma mais simples possível, porém não mais simples que o possível" Albert Einstein 


\section{NORMAS ADOTADAS}

Esta tese está de acordo com as seguintes normas, em vigor no momento desta publicação:

Referências: adaptado de International Committee of Medical Journals Editors (Vancouver)

Universidade de São Paulo. Faculdade de Medicina. Serviço de Biblioteca e Documentação. Guia de apresentação de dissertações, teses e monografias. Elaborado por Anneliese Carneiro da Cunha, Maria Julia de A. L. Freddi, Maria F.Crestana, Marinalva de Souza Aragão, Suely Campos Cardoso, Valéria Vilhena. 3a ed. São Paulo: Serviço de Biblioteca e Documentação; 2011.

Abreviaturas dos títulos dos periódicos, de acordo com List of Journals Indexed in Index Medicus. 
SUMÁRIO 
Abreviações

Lista de Tabelas e Anexos

Resumo

Summary

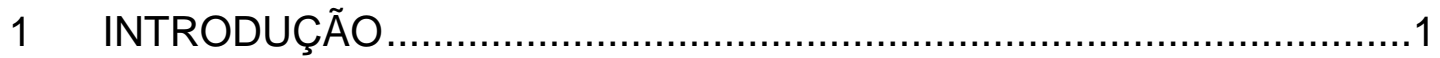

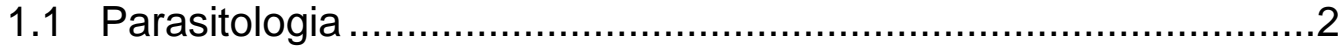

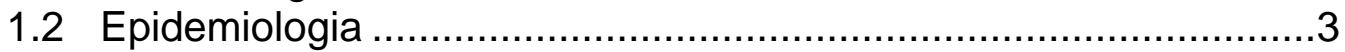

1.3 Quadro clínico....................................................................

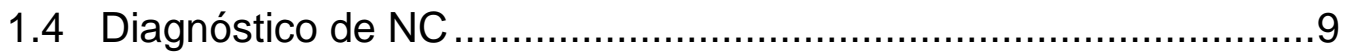

1.5 Quadro cognitivo........................................................

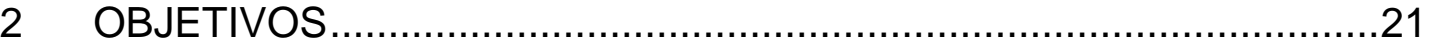

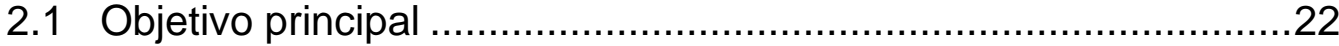

2.2 Objetivos específicos .....................................................22

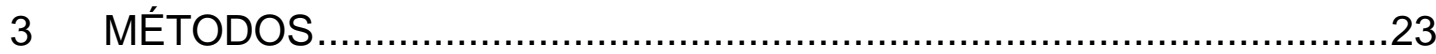

3.1 Participantes ...................................................................... 24

3.2 Pacientes com NC calcificada ..............................................26

3.3 Grupos de comparação ....................................................27

3.4 Recrutamento e emparelhamento ........................................30

3.5 Avaliação cognitiva e funcional .............................................. 30

3.6 Análise estatística ........................................................... 32

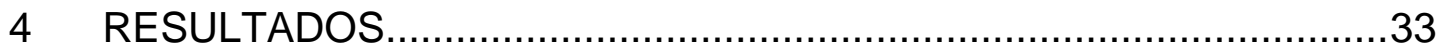

4.1 Pacientes excluídos da análise .............................................34

4.2 Presença de depressão ................................................... 34

4.3 Pacientes incluídos na análise ............................................ 35

4.4 Avaliação cognitiva ........................................................ 42

4.4.1 Análise intergrupos .............................................. 42

4.4.2 Análise intragrupos .........................................42

4.5 Análise de neuroimagem .............................................44

4.6 Análise prospectiva......................................................45

4.7 Poder estatístico do estudo ................................................46

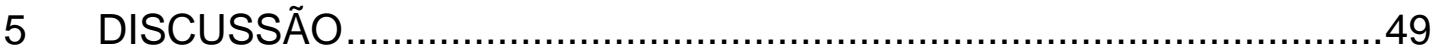

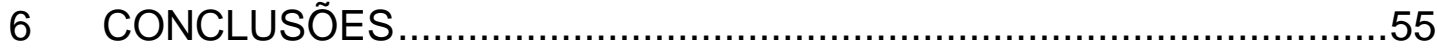

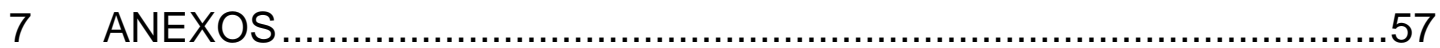

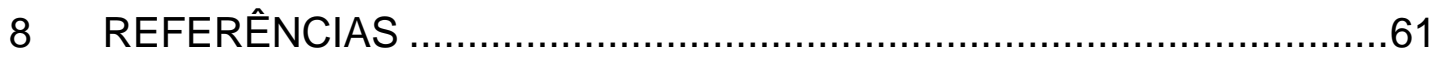


LISTAS

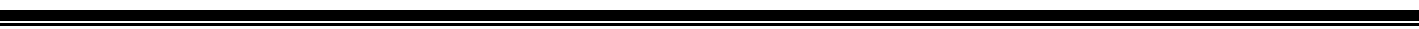




\section{Abreviações}

\begin{tabular}{|c|c|}
\hline A-NC & neurocisticercose ativa \\
\hline BBRC & Bateria Breve de Rastreio Cognitivo \\
\hline CCSD & Comprometimento Cognitivo Sem Demência \\
\hline CERAD & Consortium to Establish a Registry for Alzheimer's Disease \\
\hline $\mathrm{C}-\mathrm{NC}$ & neurocisiticercose calcificada \\
\hline CS & Controles Saudáveis \\
\hline DAE & Drogas antiepilépticas \\
\hline DR & Desenho do relógio \\
\hline DSM-IV & Diagnostic and Statistical Manual of Mental Disorders 4th Edition \\
\hline EDD & Extensão de dígitos em ordem direta \\
\hline EDI & Extensão de dígitos em ordem indireta \\
\hline EMT & Esclerose Mesial Temporal \\
\hline FVS & Teste de Fluência Verbal Semântica \\
\hline HC-FMUSP & $\begin{array}{l}\text { Hospital das Clínicas da Faculdade de Medicina da } \\
\text { Universidade de São Paulo }\end{array}$ \\
\hline LCR & Líquido cefalorraquidiano \\
\hline MEEM & MiniExame do Estado Mental \\
\hline MNV & Memória Não Verbal \\
\hline MV & Memória Verbal \\
\hline NC & Neurocisticercose \\
\hline PC & Praxia Construtiva \\
\hline QAFP & Questionário de Atividades Funcionais de Pfeffer \\
\hline RM & Ressonância magnética de crânio \\
\hline SNC & Sistema Nervoso Central \\
\hline TC & Tomografia computadorizada de crânio \\
\hline TICS & Teste de Interferência de Cores de Stroop \\
\hline $\mathrm{TN}$ & Teste de Nomeação \\
\hline TTA & Teste de Trilhas \\
\hline TTB & Teste de Trilhas B \\
\hline
\end{tabular}




\section{Lista de Tabelas e Anexos}

Tabela 1 - Apresentação dos casos quanto ao registro hospitalar, gênero, idade, naturalidade e procedência

Tabela 2 - Distribuição da idade, anos de educação e uso de drogas antiepilépticas e freqüência de crises epilépticas entre os grupos.

Tabela 3 - Frequência das alterações cognitivas encontradas nos pacientes com NC calcificada e ativa

Tabela 4 - Número total de lesões na neuroimagem de cada grupo de neurocisticercose calcificada e ativa e de seus subgrupos de acordo com as alterações cognitivas

Anexo 1 - Resultados dos testes cognitivos dos grupos C-NC, CS, A-NC.

Anexo 2 - Resultados dos testes cognitivos e funcionais de indivíduos do grupo controle saudável (CS) e o número de pacientes do grupo C-NC com escores alterados

Anexo 3 - Resultados dos testes cognitivos e funcionais de indivíduos do grupo controle saudável (CS) e o número de pacientes do grupo A-NC com escores alterados 
RESUMO 
Rodrigues CL. Espectro do comprometimento cognitivo na neurocisticercose: diferenças de acordo com a fase da doença [tese]. São Paulo: Faculdade de Medicina, Universidade de São Paulo; 2011. 69 p.

Introdução: O declínio cognitivo relacionado à neurocisticercose (NC) ainda permanece mal caracterizado e pouco diagnosticado. Recentemente, nosso grupo mostrou que uma significativa parcela de pacientes com NC na fase cística ativa apresenta-se com declínio cognitivo e comprometimento funcional (Ciampi de Andrade et al, 2010). Não existem estudos controlados que avaliem essas alterações em pacientes com NC na fase calcificada na literatura até presente momento. Objetivos: Avaliar o desempenho cognitivo do maior subgrupo de NC, constituído de pacientes com NC na fase estritamente calcificada (C-NC). Verificar a presença de demência e comprometimento cognitivo sem demência (CCSD) nesses pacientes e tentar correlacionar os resultados obtidos a achados de neuroimagem. Investigar se existe presença de um espectro de anormalidade cognitiva na NC conforme a fase da doença. Metódos e Casuística: Quarenta pacientes (média de idade $=37,6 \pm 11,3$ anos e escolaridade média $=7,0 \pm 3,5$ anos), com critérios diagnósticos absolutos de C-NC foram submetidos à avaliação cognitiva e funcional, sendo comparados a 40 pacientes controles saudáveis (CS) e 40 pacientes com NC ativa (A-NC), emparelhados por idade e nível educacional. Todos os pacientes do grupo C-NC foram submetidos a estudo de RM de encéfalo, a fim de excluir outras causas de epilepsia e sinais de atividade inflamatória. Resultados: Os doentes C-NC apresentaram uma média de 9,40 \pm 3,13 testes alterados dos 30 da bateria de avaliação cognitiva quando comparados aos CS. Nenhum paciente C-NC mostrou critérios para demência e 10 (25\%) tiveram critérios para CCSD. O grupo ANC tinha 5 pacientes (12,5\%) com demência e $11(27,5 \%)$ com CCSD. Mais de $50 \%$ dos doentes C-NC apresentaram desempenho inferior em memória verbal, atenção e função executiva em comparação aos CS. Não se encontrou correlação entre as alterações nos testes cognitivos nos pacientes $\mathrm{C}-\mathrm{NC}$ e A-NC e os achados de neuroimagem e a frequência de crises epilépticas. Dos 4 pacientes reavaliados com demência do grupo A-NC após 2 anos, 3 deles ainda mantinham critérios de demência e na reavaliação por neuroimagem ainda apresentavam cistos. O único que não mais preenchia critérios para demência ou CCSD, exibia apenas calcificações na neuroimagem controle. Conclusões: Os resultados do presente estudo controlado indicaram que a NC independente de sua fase leva a um espectro de alterações cognitivas que varia de comprometimento em um simples domínio, CCSD até, ocasionalmente, demência. Estes achados parecem ser mais intensos durante a fase ativa cística da doença e menos proeminentes no estágio calcificado.

Descritores: neurocisticercose calcificada, cognição, comprometimento cognitivo, demência. 
SUMMARY 
Rodrigues CL. The spectrum of cognitive impairment in neurocysticercosis differences according to disease phase [thesis]. São Paulo: "Faculdade de Medicina, Universidade de São Paulo"; 2011. 69 p.

Introduction: Cognitive decline related to neurocysticercosis (NC) remains poorly characterized and underdiagnosed. We have previously shown that a significant proportion of active NC patients (A-NC) present cognitive and functional impairment. Until now, there is no control study that have evaluated cognitive abnormalities in patients in the calcified phase of NC. Objective: To evaluate the cognitive performance of the largest subgroup of $\mathrm{NC}$, the strict calcified patients (C-NC). Check the presence of dementia and cognitive impairment no-dementia (CIND) and correlate the results with neuroimaging findings. To investigate whether there is a spectrum of cognitive abnormalities in the disease according to disease phase. Methods and participants: Forty treatment-naive patients with C-NC aged $37.6 \pm 11.3$ years and fulfilling absolute criteria for definitive C-NC were submitted to a comprehensive cognitive and functional evaluation and were compared with 40 active NC patients (A-NC) and 40 healthy controls (HC) matched for age and education. All patients of C-NC group underwent brain MRI study in order to exclude other causes of epilepsy and signs of inflammatory activity. Results: Patients with C-NC presented $9.4 \pm 3.1$ altered test scores out of the 30 from the cognitive battery when compared to HC. No C-NC patient had dementia and 10 patients (25\%) presented CIND. The A-NCYST group had five patients (12.5\%) with dementia and 11 patients $(27.5 \%)$ with CIND. More than $50 \%$ of C-NC patients had low performance in verbal memory, attention and executive functions in comparison to CS. No significant correlation was found between cognitive performance and the number of lesions and seizure frequency. On follow-up, three out of five previously demented A-NCYST patients still presented cystic lesions with scolex on MRI and were still demented. One patient died and the remaining patient no longer fulfilled criteria for neither dementia nor CIND, presenting exclusively calcified lesions on neuroimaging. Conclusion: The results of our controlled study shows that NC, independently of its phase, leads to a spectrum of cognitive abnormalities, ranging from impairment in a single domain, to CIND and, occasionally, to dementia. These findings are more conspicuous during active vesicular phase and less prominent in calcified stages.

Descriptors: calcified neurocysticercosis, cognition, cognitive impairment, dementia. 


\subsection{Parasitologia}

A Cistecercose humana é causada pela forma larvária do parasita Taenia solium. Ela ocorre quando o Homem, que é o único hospedeiro da forma adulta do parasita, ocupa a posição de hospedeiro intermediário no ciclo de vida do verme. No ciclo biológico natural do parasita, em razão dos hábitos coprofágicos, o suíno ingere os ovos eliminados com as fezes humanas. Uma vez ingeridos, os ovos liberam os embriões hexacantos no sistema digestivo dos suínos, que migram ativamente para a circulação sanguínea, fixando-se em tecidos de sua predileção, como o muscular, subcutâneo e o sistema nervoso central (SNC). Nesses locais, o embrião desenvolve a forma vesicular característica, o Cysticercus cellulosae, com escólex invaginado, que constitui a forma larvária da Taenia solium. Ao ingerir a carne de porco mal cozida e contaminada com cisticercos, o homem desenvolve a teníase intestinal que atinge a fase de verme adulto no intestino delgado proximal. A Taenia cresce a partir da parte proximal e desprende anéis em sua região terminal, repletos de ovos (cerca de 30 a 50 mil) que se disseminam no meio ambiente pelas fezes humanas. A ingestão dessas fezes contaminadas por ovos pelos suínos completa o ciclo de vida do parasita. 
A Cistecercose ocorre quando o homem ingere ovos de Taenia solium, desempenhando portanto, papel de hospedeiro intermediário. O fato possibilita o aparecimento da forma vesicular no sistema nervoso central (SNC). No ciclo natural, esse papel é reservado aos suínos (Canelas, 1962; Vaz, 1996; Garcia et al., 2005). A forma mais comum e grave é representada pelo comprometimento do sistema nervoso pela forma larvária da Taenia solium, denominada Neurocisticercose (NC). Cada cisticerco sobrevive cerca de 3 a 6 anos e evolui para degeneração espontânea após esse período, passando por uma fase de granuloma, com posterior calcificação (Takayanagui, 1983; Garcia et al., 2003).

\subsection{Epidemiologia}

A NC é a mais importante e frequente parasitose do SNC (Canelas, 1962), sendo bastante prevalente em países da América do Sul, Ásia e África (Roman et al., 2000; Varma et al., 2002; Agapejev, 2003; Kraft et al., 2007). No entanto, nas últimas décadas foram publicados trabalhos mostrando crescente número de casos nos Estados Unidos da América (Shandera et al., 1994; Ong et al., 2002; Townes et al., 2004; De La Garza et al., 2005; Serpa et al., 2011; Sorvillo et al., 2011) e em países da Europa (Alvarez-Rodriguez et al., 2005; Mas-Sesé et al., 2008; Ruiz et al., 2011). 
A Organização Mundial de Saúde calcula que ocorram, por ano, 50 mil óbitos em razão da NC (Roman et al., 2000). Complicações crônicas não fatais como epilepsia e síndrome de hipertensão intracraniana acometem um número maior de indivíduos e causam impacto negativo sobre a vida dos habitantes e a economia das áreas endêmicas. Em nosso meio, onde boa parte da população está exposta à Taenia solium, Agapejev mostrou prevalência de 1,5\% em estudos de necropsia (Agapejev, 2003). Em estudo retrospectivo de 63 anos de acompanhamento de pacientes no Hospital das Clínicas da Faculdade de Medicina da Universidade de São Paulo, SpinaFrança et al. encontraram prevalência 1,13\% de NC em 139.000 pacientes internados. A NC acomete tanto homens como mulheres, de qualquer raça e de todas as faixas etárias, com predomínio entre 11 e 35 anos (Takayanagui, 1990).

\subsection{Quadro Clínico}

Os aspectos clínicos da NC estão relacionados a múltiplos fatores inter-relacionados, o que confere caráter de variabilidade e imprevisibilidade clínica à doença. O quadro clínico depende da interação do parasita com os fatores de defesa do hospedeiro, variando de acordo com o tipo morfológico do cisticerco, número de cistos, sua localização e fase de desenvolvimento. 
1.3.1 Tipo morfológico: a forma Cysticercus cellulosae dá origem à forma vesicular clássica, em geral, intraparenquimatosa, sendo a mais prevalente na maioria das casuísticas publicadas. A forma racemosa (Cysticercus racemosus) é habitualmente desprovida de escólex, sendo formada por várias vesículas confluentes e localizada, frequentemente, nas cisternas da base do crânio (Garcia et al., 2005).

1.3.2. Números de cistos: pode haver desde um cisto isolado até várias dezenas de cisticercos.

1.3.3 Localização dos cistos: podem localizar-se no espaço subaracnóideo da convexidade do cérebro, nas cisternas, no parênquima (neste caso, o local mais comum é a transição córtico-subcortical), nos ventrículos e no canal raquidiano.

1.3.4 Fase de evolução biológica do parasita:

a) Estágio vesicular: a princípio, os cistos são biologicamente viáveis, apresentando-se como vesículas íntegras, sem evidência de processo inflamatório a seu redor. Isso decorre de imunossupressão local e da ativação de mecanismos de evasão imunológica (Garcia et al., 2005). O fluido vesicular é claro e o escólex, bem individualizado. O paciente tende a permanecer assintomático ou oligossintomático em razão do equilíbrio entre parasita e hospedeiro.

b) Estágio coloidal: é o momento em que os cistos sofrem degeneração, após período de 3 a 6 anos ou após tratamento com 
drogas parasiticidas. O fluido vesicular passa a se tornar turvo e o escólex, a apresentar sinais de degeneração e rotura da barreira hematoencefálica. após a exposição da superfície do parasita ao sistema imune do hospedeiro, há perda dos mecanismos de supressão. O parasita passa a apresentar cápsula espessa, o tecido fica edemaciado e há ativação astrocítica com reação glial periférica. Aos exames de neuroimagem estrutural, observa-se realce anelar à tomografia de crânio (TC) ou hipersinal em imagens ponderadas em T2 ou FLAIR ("Fluid-attenuated Inversion Recovery Image") à ressonância magnética (RM) de crânio (Takayanagui, 1983; 2001; Del Brutto et al., 2001; Lucato et al., 2007).

c) Estágio nodular: neste estágio, o conteúdo do cisto torna-se mais espesso e o escólex deixa de ser bem caracterizado. O cisto assume formato nodular, semelhante a um granuloma e há persistência de reação inflamatória perilesional.

d) Estágio calcificado: após o estágio nodular, há importante redução das dimensões da lesão, para cerca de $20 \%$ do tamanho inicial. Gradualmente, há deposição de cristais de cálcio, até que o cisto atinja a fase de grânulo calcificado.

Na literatura, o estágio calcificado é objeto de discussão. Acreditavase que a fase calcificada fosse completamente inativa, inerte, cicatricial e sem atividade antigênica. Com certa frequência, observam-se a RM e realces inflamatórios perilesionais em formas calcificadas. Esse achado 
levou alguns autores a avaliarem a possibilidade teórica de que, ao menos em sua fase inicial, esses cistos residuais possam continuar apresentando atividade antigênica. Tal hipótese explicaria a persistência de manifestações clínicas de epilepsia em alguns pacientes durante períodos variáveis após a caracterização da calcificação dos parasitas (Carpio et al., 2002; Garcia et al., 2004; Sotelo et al., 1996; 2004; Nash et al., 2004). Os granulomas cisticercóticos têm propensão peculiar para se calcificar. Em áreas endêmicas, inclusive, a presença de granulomas em estágio calcificado é o achado mais frequente na neuroimagem da NC (Cruz et al., 1999; GarciaNova et al., 2001).

Constata-se que a maioria dos estudos sobre manifestações clínicas cognitivas na NC não fornece informações quanto ao estágio de evolução dos parasitas nos pacientes avaliados, não deixando clara a porcentagem da casuística formada por pacientes com a forma calcificada inativa (cicatricial), pela forma coloidal/nodular (em degeneração) e a vesicular (oligo/assintomática) (Forlenza et al., 1997; Boppré et al., 2001; RamirezBermudez et al., 2005; Terra-Bustamante et al., 2005).

Em áreas endêmicas há grande parte da população com infestação resolvida espontaneamente e que talvez nunca tenha apresentado sintomatologia exuberante. Cerca de $20 \%$ de indivíduos assintomáticos residentes em áreas endêmicas possuem calcificação intracraniana (Roman et al., 2000; Medina et al., 2005; Garcia et al., 2005).

Assim, estudos que não explicitam o estágio da doença de seus pacientes podem estar avaliando pacientes com NC resolvida, 
completamente assintomáticos, que apresentam alterações clínicas não relacionadas à NC, e sim a outras afecções igualmente prevalentes na população geral. Por exemplo, outras causas de epilepsia, outras doenças infecciosas ou transtornos do humor e do funcionamento cognitivo não relacionados à NC.

A casuística de 276 pacientes com NC analisada por Canelas (1962) permitiu uma divisão das apresentações clínicas da doença da seguinte forma:

- Forma hipertensiva: há componente de síndrome de hipertensão intracraniana (HIC). Na maioria das vezes, é associada ao bloqueio do fluxo liquórico, estando com mais freqüência relacionada à forma racemosa da doença;

- Forma convulsiva: encontrou-se igual prevalência de crises focais e generalizadas. Esta forma foi a mais prevalente na casuística avaliada, com HIC;

- Forma com manifestações neurológicas focais ou difusas: manifestações neurológicas isoladas, não associadas a formas hipertensivas ou convulsivas, foram verificadas em menos de $10 \%$ dos pacientes; e

- Forma psíquica: embora de bem caracterizada nesta casuística, foram encontradas somente em associação a outras manifestações clínicas como HIC, crises convulsivas ou manifestações neurológicas generalizadas da doença. O autor não encontrou casos isolados de alterações psiquiátricas na casuística avaliada. 
Em estudo mais recente, as manifestações clínicas com freqüência observadas na NC foram crises epilépticas (62\%), HIC (38\%), meningite cisticercótica (35\%) e transtornos psiquiátricos (11\%). Com mais raridade, os pacientes apresentam forma apoplética ou endarterítica $(2,8 \%)$ e síndrome medular (0,5\%) (Takanayagui et al., 1983, 2001).

\subsection{Diagnóstico da NC}

Havia uma década que foram propostos critérios para o diagnóstico da NC (Del Brutto et al., 2001). Estes foram divididos em absolutos, maiores, menores e epidemiológicos. O diagnóstico é denominado definitivo ou provável com base nas combinações dos seguintes critérios:

a) Critérios absolutos:

1. Demonstração histológica do parasita por biópsia de cérebro ou medula espinhal;

2. Lesão cística com evidência de escólex em TC ou RM; e

3. Visualização sub-retiniana direta do parasita à fundoscopia.

b) Critérios maiores:

1. Lesões altamente sugestivas de NC em estudos de neuroimagem;

2. Anticorpos anticisticercos séricos positivos ("enzime-linked immunotransfer blot"-EITB - ELISA); 
3. Resolução de lesão cística intracraniana após tratamento com albendazol ou praziquantel; e

4. Resolução espontânea de lesão única pequena, que capta contraste, em exame de neuroimagem.

c) Critérios menores:

1. Lesões compatíveis com NC em estudos de neuroimagem;

2. Manifestação clínica sugestiva de NC; e

3. Reação de ELISA no LCR positiva para anticorpos anticisticerco ou antígenos de cisticercos.

d) Critérios epidemiológicos:

1. Evidência de contactante doméstico com teníase;

2. Indivíduos naturais ou provenientes de região onde NC é endêmica; e

3. História de viagem frequente para localidades em que NC é endêmica.

- Diagnóstico definitivo:

Presença de um critério absoluto; e

Presença de dois critérios maiores, um menor e um epidemiológico.

- Diagnóstico provável:

Presença de um critério maior e dois menores;

Presença de um critério maior, um menor e um epidemiológico;

Presença de três critérios menores e um epidemiológico. 
A publicação que propõe estes critérios diagnósticos faz referência a certos achados de neuroimagem relacionados à NC (Del Brutto et al., 2001). No item "Critérios maiores", os autores referem- se a lesões "altamente sugestivas de NC". Este tipo de lesão é descrito no próprio texto como:

a. Lesões císticas sem visualização de escólex;

b. Lesões realçadas com contraste, tanto anelares como nodulares, únicas ou múltiplas; e

c. Calcificações arredondadas intraparenquimatosas.

As lesões descritas nos "Critérios menores" como "compatíveis com NC" são as seguintes:

a. Hidrocefalia;

b. Realce anômalo nas leptomeninges; e

c. Mielograma evidenciando múltiplas falhas de enchimento.

Atualmente, a proposta de critérios diagnósticos é popular e bastante difundida. No entanto, pacientes bem heterogêneos podem preencher os critérios diagnósticos para NC. Isto ocorre porque tais recomendações privilegiam os dados de neuroimagem em detrimento da apresentação clínica da doença. Isso faz com que preferencialmente sejam selecionados pacientes com a forma cística da doença, deixando de lado os que apresentam manifestações clínicas da forma racemosa da NC, como HIC. Assim, ao se utilizar estes critérios acaba-se por incluir um maior número de pacientes com as formas convulsivas da NC, mais associadas a 
cistos intracranianos (Bragazza et al., 2002; Garcia et al., 2005; Montano et al., 2005).

Algumas reações sorológicas para a cisticercose humana foram desenvolvidas, muitas delas de valor limitado por suas baixas sensibilidades e especificidades. O EITB (enzyme-linked immunoelectrotransfer blot) é um teste sorológico que utiliza extratos de antígenos purificados, e tem sido recomendado para o diagnóstico da doença. Apesar de sua popularidade e hoje ser considerado critério maior para o diagnóstico, apresenta duas grandes limitações. A primeira diz respeito ao alto índice de falsa negatividade desta reação, quando o paciente apresenta uma única lesão intracraniana, situação que representa um número apreciável de casos (descrita, por exemplo, em 60\% dos casos relatados na Índia) ou quando existem apenas lesões calcificadas. A segunda limitação, relaciona-se ao fato de ser um teste obtido pela análise do soro sanguíneo e sua positividade poder estar relacionada a uma forma apenas extraneurológica da cisticercose. Outro possível fator limitante para sua aplicação é o custo relativamente alto (Abraham, 2006).

Assim, estudos que desejam aprofundar-se nas manifestações clínicas da NC não devem se restringir apenas à seleção de pacientes por "critérios diagnósticos para NC", mas atentar para o fato que mesmo dentro dos critérios acima existem pacientes em fases distintas de evolução da doença, sujeitos a diferentes complicações e de prognóstico diverso. Pesquisas sobre essa doença devem definir claramente que tipo de pacientes com NC estão sendo avaliados e particularizar seus achados para 
cada um desses diferentes subtipos. A seleção do grupo controle também deve privilegiar estas nuanças relacionadas à NC.

\subsection{Quadro Cognitivo}

Nas últimas décadas, muitas informações importantes foram acumuladas em relação à fisiopatologia, diagnóstico e tratamento da NC. Entretanto, alguns aspectos clínicos permanecem compreendidos de modo insuficiente, tais como o comprometimento cognitivo relacionado à NC. Manifestações neuropsiquiátricas foram primeiramente descritas por Griesinger (Griesinger, 1862). Nas primeiras décadas do século XIX, Tretiakoff, Pacheco e Silva descreveram alguns pacientes com "debilidade mental e fraqueza intelectual" (Tretiakoff, 1924). Em 1945, após extensa pesquisa de prontuários médicos, Dixon e Hargreaves, encontraram 5\% de pacientes com NC com importante deterioração do estado mental (Dixon et al., 1945). Mais de uma década depois, Dixon, Lipscomb detectaram 21 casos de deficiência mental $(4,6 \%)$ entre os soldados que retornavam de batalhas na Índia (Dixon et al., 1961). Embora utilizem termos inespecíficos e vagos em suas séries, tais achados, basicamente disponíveis com base nos estudos realizados em instituições para doentes mentais na primeira metade do século, trouxeram luz à possibilidade de ocorrência de demência secundária da parasitose do SNC. Canelas (1962) encontrou essas 
alterações somente associadas à HIC, déficits neurológicos focais e síndrome convulsiva.

Nas décadas de 1980 e 1990, alguns estudos foram publicados voltados especificamente para anormalidades psiquiátricas. Alterações mentais que poderiam mimetizar a esquizofrenia, transtornos afetivos maiores e demência foram relatados por Tavares Jr. (Tavares Jr, 1993). Sandyk et al. relataram o caso de um homem idoso que se apresentou com demência progressiva, inicialmente rotulada como doença de Alzheimer, no qual o diagnóstico da NC foi posteriormente demonstrado. A demência desse paciente respondeu favoravelmente ao tratamento com esteroides e mebendazol (Sandyk et al., 1987). O estudo de Agapejev (2003) chama atenção porque relata a frequência dessas alterações em 9\% a 23\% dos pacientes (Agapejev, 2003).

Alguns trabalhos mostraram que as manifestações neuropsiquiátricas da NC, incluindo demência, são mais frequentes do que se acreditava anteriormente (Forlenza et al., 1997; Ramirez-Bermudez et al., 2005). Em 1997, Forlenza et al. publicaram o primeiro estudo de analise de pacientes com diagnóstico da NC com instrumentos adequados e validados para avaliação neuropsiquiátrica. Foram analisados 38 pacientes não selecionados, consecutivamente admitidos em ambulatório especializado de hospital universitário, com faixa etária entre 18 e 60 anos, que foram classificados de acordo com a localização da lesão no SNC e também conforme a atividade da doença (ativa ou inativa). Sessenta e seis por cento deles tinham baixa escolaridade. Epilepsia foi a apresentação clínica mais 
comum encontrada em 23 pacientes (60,5\%). A NC ativa foi diagnosticada em 29 (76,3\%) pacientes. Os transtornos depressivos foram diagnosticados em 52,6\%, sendo essa uma das principais conclusões do estudo psicopatológico (Forlenza et al., 1997). A inovação deste trabalho foi a utilização de entrevistas semiestruturadas e de critérios diagnósticos validados, o que proporcionou avaliação mais confiável do estado mental da casuística.

No entanto, esta pesquisa apresentou algumas limitações metodológicas. Não foi utilizado grupo controle. Como $66 \%$ dos pacientes tinham escolaridade menor que 4anos, as alterações nos testes neuropsicológicos poderiam ser, em parte, decorrentes do nível educacional baixo. O uso de controles contornaria esta questão, pois o desempenho dos doentes com NC poderia ser comparado a controles saudáveis com escolaridade semelhante. O elevado número de indivíduos deprimidos da amostra pode ter sido em parte responsável, pelo menos em parte, pelo baixo desempenho cognitivo desses pacientes. Além disso, os dados clínicos obtidos não foram abertamente correlacionados com os achados de neuroimagem estrutural (estágios ativos ou inativos calcificados).

Dois estudos adicionais avaliaram o perfil cognitivo de pacientes com NC e epilepsia: o de Boppré et al. (2001) e o de Terra-Bustamante et al. (2005). O primeiro (Boppré et al., 2001) foi composto por dois grupos de indivíduos epilépticos a um grupo controle: um grupo de 22 pacientes com epilepsia sintomática secundária a diversas etiologias, outro grupo de 20 pacientes com epilepsia sem causa aparente (a não ser a presença de 
calcificações intracranianas) e um grupo controle, sem epilepsia, de 29 indivíduos. O estudo não encontrou diferenças entre os grupos de doentes epilépticos com e sem calcificações intracranianas sugestivas de NC. Ambos os grupos tiveram desempenho inferior ao grupo controle sem epilepsia em testes de avaliação de memória verbal, reconhecimento espacial, habilidades visuais construtivas, reconhecimento de faces famosas, abstração e julgamento. Os autores não relataram a presença de depressão nos pacientes. Estes dados foram uma valiosa contribuição ao tema. No entanto, o estudo restringiu-se a pacientes que apresentavam calcificações intracranianas associadas à epilepsia, uma população que poderia nem receber o diagnóstico de NC se os critérios atuais fossem utilizados (Del Brutto et al., 2001). O segundo estudo (Terra-Bustamante et al., 2005) avaliou uma população de pacientes com epilepsia sintomática secundária à esclerose mesial temporal (EMT) composta por portadores $(n=32)$ e não portadores $(n=48)$ de calcificações intracranianas e, da mesma forma que o anterior, não encontrou diferenças na avaliação cognitiva entre os dois grupos. No entanto, o estudo só utilizou pacientes com EMT que foram submetidos à ressecção cirúrgica, avaliando, assim, um grupo bem restrito de indivíduos. Seu maior valor talvez seja o de ampliar o conhecimento do papel de calcificações intracranianas associadas a outras causas de epilepsia sintomática e a influência desta associação no prognóstico pósoperatório dos doentes com EMT.

Ramirez-Bermudez et al. 2005, realizaram uma pesquisa com 90 pacientes com NC e procuraram avaliar se a presença de demência era 
reversível após o tratamento com albendazol e esteroides. Demência estava presente em $15,5 \%$ dos sujeitos $(n=14)$ no momento da avaliação inicial, de acordo com os critérios do DSM-IV. A análise dos dois grupos (dementes e não dementes) revelou que o grupo de pacientes com demência era mais velho, tinha menor escolaridade, proporção maior de casos com epilepsia e maior número de lesões parasitárias do que o grupo de indivíduos sem demência.

Não houve diferença significativa entre pacientes com demência e sem demência em relação à NC estar ativa ou inativa. Seis meses após a avaliação inicial, três pacientes com demência inicial $(21,5 \%)$ continuaram a ter o mesmo distúrbio, sendo dois com lesões calcificadas que não haviam recebido tratamento anteriormente. Onze pacientes $(78,5 \%)$ não mais preencheram os critérios do DSM-IV para demência, embora apenas seis desses pacientes exibiram recuperação completa. Todas as funções cognitivas avaliadas no grupo de pacientes com demência apresentaram melhora significativa após o tratamento. Os autores concluíram que a demência relacionada à NC foi mais comum em pacientes susceptíveis (com idade avançada, baixo nível educacional, epilepsia e maior número de lesões) e teve prognóstico mais favorável quando tratados com drogas antiparasitárias e esteróides. Novamente, este trabalho também não incluiu grupo controlado por placebo e o pequeno tamanho do grupo com demência torna difícil estabelecer conclusões mais precisas sobre o papel de cada fator biológico envolvido na ocorrência de demência. Além disso, os dados de correlação encontrados poderiam sugerir que a presença de demência 
estava relacionada a fatores secundários ou de confusão em lugar da presença NC propriamente dita (Ciampi de Andrade et al., 2010).

Recentemente, publicamos o primeiro estudo controlado sobre as manifestações neuropsiquiátricas da NC em 40 pacientes sem tratamento, apresentando exclusivamente NC em sua forma ativa, definida por critérios absolutos pela presença de, ao menos, um cisto com escólex íntegro à RM de crânio (Ciampi de Andrade et al., 2010). O desempenho cognitivo e funcional de pacientes com NC ativa foi comparado a um grupo de controles saudáveis (CS) devidamente emparelhados por idade e nível educacional. Ainda, com o intuito de aferir se a presença de crises epilépticas e uso de DAE poderiam influenciar o desempenho cognitivo desses pacientes, comparamos o desempenho dos pacientes com NC ativa a um grupo de pacientes com epilepsia criptogênica (EC) de similar perfil de idade e escolaridade. Cinco pacientes (12,5\% da amostra) preencheram critérios diagnósticos do DSM-IV para demência. Cerca de 45\% dos pacientes $(\mathrm{N}=18)$ apresentaram algum grau de comprometimento das funções cognitivas, porém sem prejuízo funcional, o que foi denominado comprometimento cognitivo sem demência (CCSD). Todos os pacientes do grupo NC ativa apresentaram ao menos um escore cognitivo significativamente alterado em relação ao grupo CS.

Observou-se média de 13,6 \pm 5,81 resultados de testes (ou subtestes) cognitivos alterados dentre os 30 que faziam parte da avaliação para cada indivíduo com NC ativa. Mais da metade dos pacientes apresentou escores alterados em domínios como memória, atenção, funções executivas, 
linguagem e praxia construtiva. Pacientes do grupo NC ativa com e sem CCSD não diferiram significativamente quanto à idade e escolaridade. Pacientes com e sem demência não diferiram significativamente em relação à idade, nível educacional, presença de tratamento prévio para depressão com sucesso, tipo e número de DAE, duração da doença e frequência mensal de crises epilépticas. Não se encontrou correlação significativa entre os escores dos testes cognitivos e número, fase evolutiva das lesões ou sua localização.

No estudo, observou-se que pacientes com NC forma ativa apresentaram desempenho significativamente inferior ao grupo com EC em diversos domínios cognitivos, como funções executivas, fluência verbal, memória episódica verbal e habilidades visual-espaciais, independente da idade, nível educacional, frequência de epilepsia e do uso de DAE. Uma vez que indivíduos com EC não apresentam lesões cerebrais detectáveis, concluiu-se que as diferenças encontradas eram provavelmente relacionadas às lesões da NC ou às suas complicações diretas, como edema cerebral e inflamação local.

Até recentemente, diferentemente da impressão clínica daqueles que se dedicam à assistência de doentes com NC, a totalidade dos estudos científicos considerava a NC como um grupo de doentes homogêneos, não se estratificando os doentes de acordo com suas diferentes fases evolutivas (ex. como ativa ou calcificada). Portanto, só recentemente um trabalho avaliou o impacto da NC ativa na cognição, encontrando alta prevalência de déficits cognitivos e demência nessa população de doentes (Ciampi de 
Andrade et al., 2010). Apesar de interessante e original, a avaliação de pacientes com a fase aguda da doença talvez seja uma questão menor em termos de saúde pública. Isto porque a fase calcificada é muito mais prevalente na população geral (Nash et al., 2004), sendo o denominador comum de todos os doentes com NC após a fase inicial da doença (ativa) e intermediária (forma transicional) (Roman et al., 2000, Medina et al., 2005).

Desta forma, realizamos um estudo transversal que visou a responder se pacientes com a forma mais prevalente da NC, a calcificada, apresentam alterações cognitivas e demência quando comparados a indivíduos sadios. A fim de avaliar um eventual comprometimento cognitivo espectral nos doentes com NC, comparamos as análises do grupo calcificado aos obtidos naqueles com a forma cística da doença. Foi realizada também análise prospectiva complementar, com uma reavaliação dos doentes com demência após dois anos da primeira avaliação. 
2 OBJETIVOS 


\subsection{Objetivo principal}

Avaliar o desempenho de pacientes com NC calcificada em testes de avaliação cognitiva e funcional quando comparados a controles saudáveis.

\subsection{Objetivos específicos}

1) Avaliar a frequência de demência e de comprometimento cognitivo sem demência em pacientes com NC calcificada.

2) Analisar o perfil de alterações cognitivas em pacientes com NC calcificada.

3) Investigar a correlação entre os achados de neuroimagem, os escores em testes cognitivos e a intensidade de alterações cognitivas em pacientes com NC calcificada.

4) Investigar a ocorrência de eventual comprometimento espectral do desempenho cognitivo e funcional em pacientes com NC na fase calcificada em comparação à fase ativa. 
3 MÉTODOS 
O estudo foi realizado na Clínica Neurológica do Hospital das Clínicas da Faculdade de Medicina da Universidade de São Paulo (HCFMUSP) com envolvimento direto dos Ambulatórios de Neuroinfectologia e de Neurologia Cognitiva e do Comportamento.

O projeto de pesquisa foi aprovado pela Comissão de Ética para Análise de Projetos de Pesquisa (CAPPesq) do HC-FMUSP. Todos os participantes foram claramente esclarecidos quanto aos objetivos da pesquisa e do caráter voluntário de sua participação e assinaram o Termo de Consentimento Livre e Esclarecido.

\subsection{Participantes}

Todos os participantes foram avaliados por um neurologista devidamente treinado na administração de testes cognitivos e que desconhecia a qual grupo de estudo realmente pertencia cada indivíduo. A avaliação incluiu anamnese, triagem de abuso de álcool por meio dos questionários CAGE e AUDIT (Ewing, 1984; Saunders et al., 1993) e coleta de exames laboratoriais para avaliação de causas secundárias de déficit cognitivo e demência - eletrólitos, função tireoidiana, níveis de vitamina B12 
e ácido fólico, hemograma completo e testes imunológicos para infecção pelo HIV e Sífilis (Nitrini et al., 2005; 2005).

O tipo de manifestação clínica inicial dos pacientes com NC calcificada e ativa foi registrado, conforme os dados de prontuário ou pela anamnese. Os diferentes tipos de apresentação clínica foram organizados de acordo com Canelas (1962), incluindo:

a) Crise convulsiva seguida de síndrome epiléptica sintomática;

b) Síndrome de HIC: definida pela síndrome clínica (cefaleia, náuseas e vômitos, papiledema, alteração do nível de consciência);

c) Crise epiléptica sintomática aguda;

d) Déficit neurológico focal; e

e) Cefaleia isolada sem sinal clínico de HIC.

A duração da doença em meses e a principal síndrome clínica atual também foram registradas.

Indivíduos do grupo CS (descrição abaixo), que apresentassem alterações na triagem de abuso de álcool ou nos exames sanguíneos, foram excluídos do estudo. A presença de altos níveis de colesterol, hipertensão arterial sistêmica, diabetes mellitus, disfunção tireoidiana, sintomas depressivos, acidente vascular encefálico e antecedente de traumatismo craniano foi investigada em todos os participantes. 


\subsection{Pacientes com NC calcificada}

Pacientes com idade inferior a 60 anos, sem tratamento antiparasitário específico documentado prévio com, pelo menos, uma lesão calcificada arredondada intraparequimatosa à TC de crânio foram incluídos no grupo NC forma calcificada (grupo C-NC). Todos esses pacientes, em algum momento da evolução doença, preencheram critérios definitivos de NC (um critério absoluto ou dois critérios maiores + um menor + um epidemiológico), segundo Del Bruto et al. (2001), estando em sua forma calcificada "inativa" (Sotelo et al., 1985) à época da avaliação cognitiva e funcional, portanto sem lesões císticas. O preenchimento de critérios definitivos foi documentada em todos os doentes de forma direta, ou por seguimento no próprio serviço ou por avaliação presencial de exames de imagem e de provas de líquor cefalorraquidiano realizados previamente. A fim de excluir outras causas de epilepsia (Terra-Bustamante et al., 2005) ou a presença de sinais inflamatórios-realce perilseional (Poeschl et al., 2006), os pacientes do grupo C-NC foram submetidos a estudo de RM de crânio no período compreendido entre 15 dias antes até 15 dias depois da avaliação. As imagens foram avaliadas por neurorradiologistas que desconheciam os resultados dos testes cognitivos dos pacientes (Lucato et al., 2007). 


\subsection{Grupos de comparação}

Indivíduos saudáveis (grupo CS) foram recrutados entre os funcionários de nossa instituição e parentes de pacientes hospitalizados em outras enfermarias do hospital. Para serem incluídos, deveriam ter idade inferior a 60 anos, ausência de antecedentes clínicos de epilepsia, de doenças infecciosas ou parasitárias intestinais crônicas ou de doença neurológica, além de exame de TC de crânio sem anormalidades significativas, especialmente, a presença de calcificações patológicas. As análises de neuroimagem foram realizadas por um neurologista e por dois neurorradiologistas que desconheciam os resultados dos exames cognitivos e de avaliação funcional.

Pacientes com idade inferior a 60 anos, sem tratamento específico, preenchendo critérios definitivos de NC em sua forma ativa (Sotelo et al., 1985) foram incluídos no grupo NC forma ativa (grupo A-NC). A NC ativa foi diagnosticada por meio de critérios absolutos, conforme Del Bruto et al. (2001), ou seja, pela presença de ao menos uma lesão cística com escólex à RM de crânio. Assim, um primeiro exame de RM foi utilizado para determinar a presença de NC em sua forma ativa. Esse mesmo exame permitiu a aferição da carga de lesões de NC pela contagem do número de lesões, seu tipo (cisto com ou sem escólex, cisto com edema, cistos transicionais, lesões calcificadas e lesões racemosas), localização geral (hemisfério direito/esquerdo, cortical/subcortical, ventrículo lateral/terceiro/quarto ou 
fossa posterior) e lobo cerebral acometido (frontal, parietal, temporal ou occipital).

As imagens foram avaliadas por neurorradiologistas que desconheciam os resultados dos testes cognitivos dos pacientes (Lucato et al., 2007). Como na maior parte dos casos, a avaliação cognitiva não foi realizada no mesmo dia da visita de inclusão, mas, sim, algumas semanas ou, eventualmente, alguns meses após, todos os pacientes foram submetidos a uma segunda RM ou TC de crânio 15 dias antes ou 15 após a avaliação cognitiva, com o intuito de se confirmar que uma lesão com escólex ainda estava presente no momento da avaliação cognitiva (RamirezBermudez et al., 2005).

Dessa forma, ficou assegurado que não havia ocorrido degeneração de cistos naquele período, o que poderia influenciar o desempenho cognitivo pela presença de delirium ou encefalopatia (Forlenza et al., 1999). Parte dos resultados obtidos com base nesse grupo, quando estes foram comparados ao desempenho de pacientes com epilepsia criptogênica $(\mathrm{N}=28)$ e de controles saudáveis ( $N=49$ ), foi recentemente publicada (Ciampi de Andrade et al., 2010).

Todos os participantes do estudo foram avaliados quanto à presença de depressão, conforme os critérios do DSM-IV. Indivíduos deprimidos do grupo CS foram excluídos do estudo. Aqueles dos grupos C-NC e A-NC foram inicialmente tratados com fluoxetina $20 \mathrm{mg}$ ao dia e reavaliados em consultas mensais. Participantes com depressão só foram submetidos à 
avaliação cognitiva, uma vez que se apresentassem assintomáticos por um período mínimo de 2 meses consecutivos.

Os pacientes dos grupos C-NC e A-NC (tanto aqueles originalmente sem depressão como os que responderam a seu tratamento) foram avaliados somente se apresentassem:

1) Ausência de crises epilépticas por, ao menos 2 semanas precedendo a avaliação cognitiva;

2) Tratamento em dose fixa de DAE por,ao menos, 1 mês precedendo a avaliação;

3) Ausência de sinais clínicos de intoxicação de DAE (níveis séricos de DAE foram mensurados em casos de suspeita clínica); e

4) Ausência de sinais de delirium, conforme uma escala de avaliação específica (Delirium Rating Scale; Trzepacz et al, 1988).

Pacientes que não preenchiam todos estes critérios tiveram sua avaliação cognitiva adiada por 2 meses. Pacientes do grupo A-NC incluídos nessa situação realizaram um terceiro exame de RM com o intuito de excluir a presença de cistos em degeneração. 


\subsection{Recrutamento e emparelhamento}

Com base em estudos prévios, considerou-se que 40 pacientes com NC de cada grupo seriam suficientes para testar nossa hipótese (Forlenza et al., 1999; Ramirez-Bermudez et al., 2005; Ciampi de Andrade et al., 2010).

O emparelhamento foi realizado em blocos de dez pacientes: para cada dez pacientes com C-NC incluídos, idade e nível educacional foram estratificados, sendo que esta informação foi usada para o recrutamento do próximo bloco de dez indivíduos do grupo CS. As estratificações por idade e nível educacional tiveram intervalos de 5 e 4 anos, respectivamente. Houve também emparelhamento desses dois grupos com o grupo A-NC.

\subsection{Avaliação cognitiva e funcional}

Todos os participantes foram submetidos à avaliação cognitiva com os seguintes instrumentos: Miniexame do Estado Mental (MEEM) (Folstein et al., 1975; Brucki et al., 2003), extensão de dígitos em ordem direta (EDD) e inversa (EDI) (Wechsler, 1981), desenho do relógio (DR), evocação da lista de dez palavras (memória verbal - MV), evocação teste de fluência verbal semântica (FVS), teste de nomeação (TN) e teste de trilhas A (TTA) e B (TTB) da "Comprehensive Battery of the Consortium to Establish a Registry of Alzheimer's Disease" (CERAD) (Bertolucc et al., 1998), praxia construtiva 
do CERAD (PC) e evocação da praxia construtiva do CERAD (Morris et al., 1989; Bertolucci et al., 2001); Bateria Breve de Rastreio Cognitivo (BBRC) (Nitrini et al., 1994; 2004); e o teste de interferência de cores de Stroop (TICS) (Stroop, 1935).

O desempenho funcional foi avaliado pelo Questionário de Atividades Funcionais de Pfeffer (QAFP; Pfeffer, 1982), preenchido baseado na entrevista com um acompanhante ou familiar próximo que tivesse contato frequente com o indivíduo. Escores acima de cinco foram considerados como indicativos de comprometimento funcional.

Demência foi diagnosticada de acordo com os critérios do DSM-IV, que foram operacionalizados da seguinte forma (Ciampi de Andrade, 2010):

1) Escores abaixo do Intervalo de Confiança (IC) $95 \%$ dos valores obtidos pelo grupo CS para os testes de evocação tardia da bateria do CERAD (MV) e da BBRC;

2) Escores abaixo do IC 95\% dos valores obtidos pelo grupo CS em pelo menos um teste adicional relacionado a um segundo domínio cognitivo além da memória;

3) Comprometimento funcional conforme a a pontuação no QAFP acima de cinco pontos.

Comprometimento cognitivo sem demência (CCSD) foi diagnosticado nos pacientes preenchendo todos os critérios de demência acima, com exceção de prejuízo funcional avaliado pelo QAFP (Ciampi de Andrade, 2010). 
Os pacientes com critérios para demência foram reavaliados após 2 anos usando o mesmo protocolo.

\subsection{Análise estatística}

Após avaliação inicial pelo teste de Shapiro-Wilk (para aferir a não normalidade da distribuição), dados dos três grupos foram comparados pelo teste não paramétrico de Kruskal-Wallis, seguido pelo teste de MannWhitney (comparação grupo a grupo), sempre que indicado. O teste de Spearman foi utilizado para as análises de correlação. Análises de regressão múltipla foram feitas para avaliar variáveis independentes potencialmente relacionadas ou não ao desempenho cognitivo. Escores cognitivos de cada paciente C-NC foram comparados com os do grupo CS. Resultados abaixo do intervalo de confiança de 95\% (nível de significância: $\alpha=5 \%$ ) foram considerados alterados, a não ser pelos testes cronometrados (TTA, TTB, e TICS), em que pontuações acima de IC 95\% foram consideradas anormais. Variáveis categoriais foram comparadas por meio do Teste Exato de Fisher. As análises inferenciais e correlações foram realizadas com o programa "SPSS Statistics 17" (Statistical Package for the Social Science) e "Minitab16". A análise para o cálculo do poder do estudo foi realizada com os escores do MEEM, por se tratar de um teste de avaliação cognitiva global (multidimensional), comparando-se os grupos C-NC e CS. Para o cálculo do poder do estudo, utilizou-se o programa Minitab 16 (Pensilvânia, EUA). 
4 RESULTADOS 


\subsection{Pacientes excluídos da análise}

Inicialmente, foram selecionados 44 pacientes para o grupo C-NC. No entanto, quatro pacientes não foram submetidos à avaliação cognitiva em razão da presença de depressão refratária ao tratamento sintomático $(n=2)$, transtorno psicótico crônico prévio $(n=1)$ e gliose frontal pós-operatória $(n=1)$. Para o grupo A-NC, foram inicialmente selecionados 45 pacientes. Destes, 5 não foram submetidos à avaliação cognitiva pela presença de retardo mental $(n=1)$, depressão refratária $(n=1)$ e por terem apresentado degeneração do último cisto íntegro com escólex no intervalo de tempo entre a primeira RM (de inclusão) e a segunda, realizada próxima da avaliação cognitiva e, desta forma, não mais apresentando NC ativa definida por critérios absolutos $(n=3)$.

\subsection{Presença de depressão}

No grupo C-NC, depressão esteve presente inicialmente em 12 pacientes. Após tratamento sintomático com inibidores seletivos da recaptação de serotonina, 10 pacientes (83,3\%) tornaram-se assintomáticos e não mais preencheram os critérios do DSM-IV para depressão. Isso ocorreu, em média, após 2 meses e meio de tratamento (20mg ao dia de 
fluoxetina). Assim, 40 pacientes do grupo C-NC, sem sintomas de depressão e sem tratamento específico documentados para NC foram submetidos à avaliação cognitiva e funcional. Portanto, destes 40 pacientes, dez necessitaram de tratamento prévio para depressão antes da avaliação (25,0\% da amostra).

No grupo A-NC, depressão esteve presente em 11 pacientes; foi tratada com sucesso em $10(90,9 \%)$ deles. Assim, 40 pacientes do grupo ANC sem tratamento antiparasitário específico para NC foram submetidos à avaliação cognitiva e funcional. Destes 40 pacientes A-NC, dez necessitaram de tratamento prévio para depressão antes da avaliação (25,0\% da amostra).

\subsection{Pacientes incluídos na análise}

Quarenta pacientes com NC forma calcificada (grupo C-NC), 40 pacientes com NC forma ativa (grupo A-NC) e 40 indivíduos controles saudáveis (grupo CS) foram submetidos à avaliação cognitiva, funcional e de neuroimagem completa, sendo assim incluídos na análise estatística. As características gerais dos doentes, incluindo registro hospitalar, idade, gênero, naturalidade e procedência constam dos dados da TABELA 1. Os pacientes do grupo C-NC (1-40) tiveram como origem os Estados do Nordeste (50\%), Sudeste (37.5\%) e Sul (12.5\%). Por ocasião do estudo, 95\% dos participantes residiam no Estado de São Paulo. Os pacientes do 
grupo A-NC (41-80) tiveram como origem Estados das Regiões Nordeste $(62,5 \%)$, Sudeste $(25 \%)$ e Sul (2,5\%). Por ocasião do estudo, $90 \%$ dos participantes residiam no Estado de São Paulo. Em relação ao gênero, $47,5 \%$ dos doentes do grupo C-NC eram do sexo masculino e $42,5 \%$ do sexo feminino e $62,5 \%$ dos doentes do grupo A-NC eram do sexo masculino e $37,5 \%$ do sexo feminino.

Participantes dos três grupos (C-NC, A-NC e CS) apresentaram semelhanças em relação à distribuição por idade $(37,63 \pm 11,35 ; 39,25 \pm$ 10,50; 40,30 $\pm 12,22$ anos; respectivamente; $p=0,600)$ e nível educacional anos de escolaridade $(7,03 \pm 3,59 ; 6,20 \pm 4,03 ; 7,18 \pm 3,68$ anos; respectivamente; $p=0,395)$ (TABELA 2).

No grupo C-NC, excluindo-se os resultados da avaliação cognitiva, a manifestação clínica inicial mais frequente foi epilepsia isolada (62,5\%), epilepsia com cefaleia associada (12,5\%), epilepsia com déficit focal $(2,5 \%)$, epilepsia com síndrome clínica de HIC (2,5\%), crise sintomática aguda isolada (10\%), cefaleia isolada sem sinais de HIC ou crise sintomática aguda (7,5\%), crise sintomática aguda associada com meningite $(2,5 \%)$, crise sintomática aguda com cefaleia sem meningite (2,5\%). Portanto, 80,0\% dos pacientes tiveram diagnóstico de epilepsia e 15\% tinham histórico de pelo menos uma crise isolada, perfazendo o total de $95 \%(n=38)$ de pacientes com histórico de crise epiléptica associada à NC. Desses 38 pacientes, cinco apresentavam crises epilépticas mensais ( $12,5 \%$ do total da amostra), outros cinco mostravam crises epilépticas semestrais e três tinham crises epilépticas anuais. Portanto, $67,5 \%$ dos pacientes do grupo C-NC 
apresentavam crises bem controladas (chegavam a ficar mais de um ano sem crise). A frequência mensal de crises epilépticas do grupo C-NC como um todo $(n=40)$ foi $0,22 \pm 0,36$ crises. Trinta e seis pacientes $(90,0 \%$ do total) estavam tomando antiepilépticos no momento avaliação, destes $80,5 \%$ usavam apenas uma medicação para controle das crises e o restante $(19,5 \%)$ usavam duas medicações. As drogas antiepilépticas mais utilizadas foram carbamazepina (39,0\%), fenitoína (25,0\%), fenobarbital (15,5\%), carbamazepina e fenobarbital (8,4\%), fenitoína e fenobarbital $(5,6 \%)$, ácido valproico (2,8\%) e oxcarbazepina (2,8\%). Na época da avaliação cognitiva, apenas $5 \%$ dos pacientes $(n=2)$ encontravam-se totalmente assintomáticos, sem queixas clínicas atuais e sem necessidade de uso de qualquer medicação. O tempo médio entre o primeiro sintoma de NC e a data da avaliação cognitiva foi de 9 anos e 8meses.

No grupo A-NC, excluindo-se os resultados da avaliação cognitiva, a manifestação clínica inicial mais frequente foi epilepsia $(67,5 \%)$, HIC (12,5\%), crise sintomática aguda $(10,0 \%)$, déficit neurológico focal $(7,5 \%)$ e cefaleia isolada sem sinais de HIC (2,5\%). Todos os pacientes incluídos no estudo apresentavam síndrome epiléptica sintomática no momento da avaliação; 10 pacientes $(25,0 \%)$ apresentaram mais de uma manifestação clínica. O tempo médio decorrido entre o primeiro sintoma de $\mathrm{NC}$ e a avaliação cognitiva foi de 7 anos e 3 meses. Pacientes do grupo A-NC apresentaram frequência mensal de crises epilépticas de 5,15 $\pm 2,63$, estatisticamente superior à frequência de crises do grupo C-NC $(p<0,001)$. A distribuição do tipo de droga antiepiléptica (DAE) utilizada por integrantes do 
grupo A-NC ocorreu da seguinte forma: carbamazepina $(62,5 \%)$, fenobarbital $(25,8 \%)$ e fenitoína $(18,7 \%)$. Estes resultados não foram estatisticamente diferentes em comparação com o uso das três medicações no grupo C-NC $(p=0,082 ; p=0,939$ e $p=0,388 ;$ respectivamente).

Níveis de colesterol, presença de hipertensão arterial sistêmica, diabetes mellitus, disfunção tireoidiana, antecedentes de depressão e acidente vascular encefálico foram semelhantes entre os integrantes dos dois grupos ( $p>0,3$ para todos os testes). Nenhum paciente dos grupos CNC e A-NC apresentou escores alterados nos questionários CAGE e AUDIT. 
Tabela 1 - Apresentação dos casos quanto ao registro hospitalar, gênero, idade, naturalidade e procedência

\begin{tabular}{|c|c|c|c|c|c|}
\hline Paciente & $\begin{array}{c}\text { Registro } \\
\text { hospitalar }\end{array}$ & Gênero & Idade & Naturalidade & Procedência \\
\hline 1 & $13488551 \mathrm{~K}$ & $M$ & 36 & MG & $\mathrm{SP}$ \\
\hline 2 & 13496292C & M & 45 & PR & SP \\
\hline 3 & $13515049 \mathrm{~J}$ & M & 45 & CE & SP \\
\hline 4 & $13534864 C$ & M & 19 & RJ & SP \\
\hline 5 & $13531996 \mathrm{C}$ & $M$ & 36 & PR & SP \\
\hline 6 & 13638990K & $M$ & 29 & CE & SP \\
\hline 7 & $13640948 \mathrm{H}$ & $M$ & 57 & RN & SP \\
\hline 8 & $\mathrm{HC}$ externo & $M$ & 23 & CE & SP \\
\hline 9 & $13674042 A$ & $\mathrm{~F}$ & 51 & SP & SP \\
\hline 10 & 13682837D & M & 45 & BA & SP \\
\hline 11 & $13686644 \mathrm{C}$ & M & 27 & BA & SP \\
\hline 12 & 13738595D & $\mathrm{F}$ & 17 & SP & SP \\
\hline 13 & $13760450 \mathrm{C}$ & $\mathrm{F}$ & 41 & $\mathrm{PI}$ & SP \\
\hline 14 & 13702468G & $M$ & 48 & MG & SP \\
\hline 15 & $\mathrm{HC}$ externo & $\mathrm{F}$ & 31 & CE & SP \\
\hline 16 & 13710822B & $\mathrm{F}$ & 23 & BA & SP \\
\hline 17 & $2430723 K$ & $\mathrm{~F}$ & 34 & CE & SP \\
\hline 18 & $2803610 G$ & $\mathrm{~F}$ & 19 & BA & BA \\
\hline 19 & $2839457 C$ & M & 24 & BA & SP \\
\hline 20 & $2865957 G$ & $\mathrm{~F}$ & 52 & PR & PR \\
\hline 21 & $3057799 A$ & $\mathrm{~F}$ & 46 & MG & SP \\
\hline 22 & $3177615 \mathrm{C}$ & M & 16 & CE & SP \\
\hline 23 & 3183910J & $M$ & 44 & PR & SP \\
\hline 24 & $3208463 F$ & $\mathrm{~F}$ & 46 & PB & SP \\
\hline 25 & $3226409 E$ & M & 27 & SP & SP \\
\hline 26 & 3244785D & $\mathrm{F}$ & 55 & SP & SP \\
\hline 27 & $3254445 \mathrm{~K}$ & $\mathrm{~F}$ & 55 & SP & SP \\
\hline 28 & 3294110B & $\mathrm{M}$ & 39 & BA & SP \\
\hline 29 & $3317594 \mathrm{~F}$ & $\mathrm{M}$ & 41 & MG & MG \\
\hline 30 & $2514919 G$ & M & 43 & PE & SP \\
\hline 31 & $3351118 \mathrm{D}$ & $\mathrm{F}$ & 42 & CE & SP \\
\hline 32 & 2911788D & M & 29 & SP & SP \\
\hline 33 & $2514920 G$ & $\mathrm{~F}$ & 35 & SP & SP \\
\hline 34 & 5170225B & F & 32 & SP & SP \\
\hline 35 & $2631659 \mathrm{C}$ & $M$ & 33 & PR & SP \\
\hline 36 & $3183910 \mathrm{~J}$ & M & 43 & BA & SP \\
\hline 37 & $3295557 F$ & M & 48 & PR & SP \\
\hline 38 & $\mathrm{HC}$ externo & $\mathrm{F}$ & 36 & CE & $\begin{array}{l}\mathrm{SP} \\
\text { continua }\end{array}$ \\
\hline
\end{tabular}


Tabela 1 - Apresentação dos casos quanto ao registro hospitalar, gênero, idade, naturalidade e procedência (conclusão)

\begin{tabular}{|c|c|c|c|c|c|}
\hline Paciente & $\begin{array}{c}\text { Registro } \\
\text { hospitalar }\end{array}$ & Gênero & Idade & Naturalidade & Procedência \\
\hline 39 & $3295557 \mathrm{~F}$ & $M$ & 55 & $\mathrm{SP}$ & $\mathrm{SP}$ \\
\hline 40 & $3093915 F$ & $\mathrm{~F}$ & 38 & SP & SP \\
\hline 41 & $13453854 \mathrm{~F}$ & $\mathrm{~F}$ & 32 & CE & SP \\
\hline 42 & 13653854G & M & 28 & BA & BA \\
\hline 43 & $13488551 \mathrm{~K}$ & $\mathrm{~F}$ & 57 & $\mathrm{BA}$ & SP \\
\hline 44 & $13496292 \mathrm{C}$ & $\mathrm{F}$ & 40 & BA & SP \\
\hline 45 & $13515049 \mathrm{~J}$ & $M$ & 43 & MG & SP \\
\hline 46 & $13625830 \mathrm{~J}$ & $M$ & 32 & SP & SP \\
\hline 47 & $13531996 C$ & $\mathrm{~F}$ & 31 & SP & SP \\
\hline 48 & $13638990 \mathrm{~K}$ & M & 47 & CE & SP \\
\hline 49 & $13640948 \mathrm{H}$ & M & 53 & MG & MG \\
\hline 50 & $13686644 C$ & $M$ & 24 & MG & SP \\
\hline 51 & $13738595 d$ & M & 58 & BA & SP \\
\hline 52 & 13702468G & M & 31 & BA & SP \\
\hline 53 & 13710822B & $\mathrm{F}$ & 30 & SP & SP \\
\hline 54 & $2803610 G$ & $\mathrm{~F}$ & 30 & MG & SP \\
\hline 55 & $2839457 C$ & $M$ & 56 & BA & BA \\
\hline 56 & $3057799 A$ & $M$ & 42 & PR & SP \\
\hline 57 & $3177615 \mathrm{C}$ & M & 52 & BA & SP \\
\hline 58 & 3183910J & $M$ & 40 & BA & SP \\
\hline 59 & $3208463 F$ & M & 33 & SE & SP \\
\hline 60 & 3244785D & $M$ & 29 & MG & SP \\
\hline 61 & $3254445 \mathrm{~K}$ & M & 38 & MG & SP \\
\hline 62 & 3294110B & M & 48 & BA & SP \\
\hline 63 & 3376573D & $\mathrm{F}$ & 31 & BA & SP \\
\hline 64 & $7048204 \mathrm{~K}$ & $\mathrm{~F}$ & 48 & SP & SP \\
\hline 65 & $7028456 I$ & $\mathrm{~F}$ & 43 & BA & SP \\
\hline 66 & $13643853 \mathrm{~J}$ & $\mathrm{~F}$ & 58 & BA & SP \\
\hline 67 & $13708373 E$ & M & 32 & CE & SP \\
\hline 68 & $13674055 \mathrm{~F}$ & $\mathrm{~F}$ & 25 & CE & SP \\
\hline 69 & $3117657 \mathrm{C}$ & $\mathrm{F}$ & 58 & MG & SP \\
\hline 70 & 44107097B & $\mathrm{F}$ & 27 & BA & SP \\
\hline 71 & $13721987 \mathrm{H}$ & M & 30 & BA & SP \\
\hline 72 & 13689100B & $\mathrm{F}$ & 49 & PE & SP \\
\hline 73 & 13719599G & $\mathrm{F}$ & 47 & SP & SP \\
\hline 74 & HC externo & M & 48 & MG & MG \\
\hline 75 & $13563857 \mathrm{~A}$ & M & 37 & BA & SP \\
\hline 76 & 3202207B & M & 36 & SP & SP \\
\hline 77 & $13536130 \mathrm{~A}$ & M & 37 & BA & SP \\
\hline 78 & $13730487 \mathrm{E}$ & M & 25 & SP & SP \\
\hline 79 & $2549364 \mathrm{~K}$ & M & 36 & SP & SP \\
\hline 80 & $2745212 \mathrm{E}$ & $\mathrm{M}$ & 29 & $\mathrm{RJ}$ & SP \\
\hline
\end{tabular}


Tabela 2 - Distribuição da idade, anos de educação e uso de drogas antiepilépticas e freqüência de crises epilépticas entre os grupos

\begin{tabular}{|c|c|c|c|c|c|c|c|c|c|c|c|c|c|}
\hline & \multicolumn{4}{|c|}{ idade } & \multicolumn{5}{|c|}{ anos de educação } & \multirow{2}{*}{ CBZ } & \multirow{2}{*}{ PHT } & \multirow{2}{*}{ FB } & \multirow{2}{*}{$\begin{array}{c}\text { crises I } \\
\text { mês. }\end{array}$} \\
\hline & $18-30$ & $31-40$ & $41-50$ & $51-60$ & analfabeto & 1 a 4 & 5 a 8 & 8 a 12 & $>12$ & & & & \\
\hline C-NC & $11(27,5)$ & $12(30 \%)$ & $11(27,5 \%)$ & $5(17 \%)$ & $1(2,5 \%)$ & $11(27,5 \%)$ & $13(32,5 \%)$ & $14(35 \%)$ & $1(2,5 \%)$ & $47,2 \%$ & $33,3 \%$ & $25 \%$ & $0,22 \pm 0,36$ \\
\hline CS & $9(22,5 \%)$ & $12(30 \%)$ & $9(22,5 \%)$ & $10(25 \%)$ & $1(2,5 \%)$ & $14(35 \%)$ & $8(20 \%)$ & $16(40 \%)$ & $1(2,5 \%)$ & - & - & - & - \\
\hline A-NC & $11(27,5 \%)$ & $13(32,5 \%)$ & $9(22,5 \%)$ & $7(17,5 \%)$ & $2(5 \%)$ & $16(40 \%)$ & $10(25 \%)$ & $10(25 \%)$ & $2(5 \%)$ & $62,5 \%$ & $18,7 \%$ & $25,8 \%$ & $5,15 \pm 2,63^{*}$ \\
\hline
\end{tabular}

LEGENDA: C-NC: neurocisticercose calcificada, CS: controles saudáveis; A-NC: neurocisticercose ativa, CBZ: Carbamazepina, PHT: Fenitoína, FB: Fenobarbital. $\left(^{*}\right) p<0,001$ 


\subsection{Avaliação cognitiva}

\subsubsection{Análise intergrupos}

O grupo C-NC teve desempenho sem diferença estatisticamente significativa ao grupo CS na avaliação cognitiva em todos os testes e subtestes avaliados. Por sua vez, o grupo A-NC apresentou desempenho significativamente inferior em comparação ao grupo C-NC nos testes cognitivos que avaliaram linguagem (TN), fluência verbal semântica (FVS) e memória não verbal (evocação tardia da BBRC e evocação tardia da praxia construtiva do CERAD). O grupo A-NC apresentou desempenho significativamente inferior ao grupo CS nos testes cognitivos que avaliaram funções executivas (TTA, TTB e TICS), memória verbal e não verbal (MV, BBRC, evocação da PC do CERAD), fluência verbal semântica (FVS) e linguagem (TN, FVS) (ANEXO 1).

\subsubsection{Análise intragrupos}

Individualmente, todos os pacientes do grupo C-NC apresentaram, ao menos, um escore cognitivo significativamente alterado. Observou-se média de 9,40 $\pm 3,13$ testes (ou subtestes) cognitivos alterados dentre os 30 que faziam parte da avaliação para cada indivíduo com NC calcificada. Metade ou mais dos pacientes do grupo C-NC apresentou escores alterados em domínios como memória verbal (MV), atenção (EDD, EDI) e funções 
executivas (EDI, TICS) (ANEXO 2). Nenhum paciente do grupo C-NC apresentou comprometimento funcional (QAFP>5). Portanto, nenhum paciente do grupo C-NC preencheu critérios préestabelecidos para demência. No entanto, um total de 10 pacientes (25\% da amostra) apresentou comprometimento concomitante da MV, da MNV e de, pelo menos, outro teste cognitivo, caracterizando a presença de CCSD.

Pacientes do grupo C-NC com e sem CCSD diferiram significativamente quanto ao número de testes cognitivos alterados por paciente $(12,40 \pm 3,13$ e 8,40 $\pm 2,57$, respectivamente, $p=0,001)$ e quanto à média de idade $(44,70 \pm 6,07$ e 35,27 $\pm 11,79 ;$ respectivamente; $p=0,022)$. No entanto, não diferiram significativamente quanto à escolaridade $(5,30 \pm 3,23$ e 7,6 $\pm 3,27$; respectivamente; $p=0,089$ ), frequência de crises epilépticas $(0,67 \pm 1,22$ e $0,38 \pm 0,77$, respectivamente; $p=0,607)$, presença de tratamento com sucesso para depressão $(0,40 \pm 0,52$ e 0,20 40,41 , respectivamente, $p=0,212)$ e tempo de diagnóstico $(13,40 \pm 10,97$ e 8,33 \pm 6,17 anos, respectivamente; $p=0,165)$.

No grupo A-NC, todos os pacientes apresentaram ao menos um escore cognitivo significativamente alterado (valores inferiores a dois desvios-padrão-IC de 95\%) em relação aos pacientes do grupo CS. Observou-se média de 11,62 \pm 3,88 testes (ou subtestes) cognitivos alterados dentre os 30 que faziam parte da avaliação para cada indivíduo com NC ativa. Este valor foi significativamente superior ao encontrado no grupo C-NC $(p=0,012)$. Metade ou mais pacientes do grupo A-NC apresentou escores alterados em domínios como memória (MV, evocação 
tardia da PC do CERAD), atenção (EDD, EDI), linguagem (TN, FVS), fluência verbal semântica (FVS) e funções executivas (EDI) (ANEXO 3). O diagnóstico de demência foi preenchido por 5 (12,5\%) pacientes com NC ativa. Pacientes com e sem demência não diferiram significativamente em relação à idade $(44,4 \pm 10,0$ e 38,5 \pm 5,4 anos; $p=0,26)$, nível educacional $(4,2 \pm 3,9$ e $6,2 \pm 3,6$ anos; $p=0,23)$, presença de tratamento prévio para depressão com sucesso $(20,0 \%$ e $28,0 \% ; p=0,97)$ e duração da doença $(8,6 \pm 7,0$ e $7,1 \pm 6,0$ anos; $p=0,52)$. Um total de 11 pacientes do grupo ANC (27,5\% da amostra) apresentaram comprometimento concomitante da MV, da MNV e de, pelo menos, outro teste cognitivo, porém sem comprometimento funcional, caracterizando diagnóstico de CCSD (TABELA 3).

Não encontramos correlação entre idade, escolaridade, frequência de crises, número de DAE e o número de testes alterados de cada paciente dos grupos C-NC e A-NC, quando analisados em separado ou agrupados (total de 80 pacientes).

\subsection{Análise de neuroimagem}

O grupo C-NC apresentou número médio total de lesões significativamente inferior ao grupo A-NC $(8,80 \pm 3,20$ e 18,73 $\pm 23,35$, respectivamente, $p=0,001)$. Pacientes do grupo C-NC com e sem CCSD não se diferenciaram estatisticamente quanto ao número total de lesões $(10,70 \pm$ 
$16,16$ e $8,17 \pm 12,35$, respectivamente, $p=0,36)$. O mesmo ocorreu com os pacientes do grupo A-NC com e sem demência (12,6 \pm 7,2 e 19,6 \pm 24,7, respectivamente, $p=0,86)$ (TABELA 4).

Não se encontrou correlação significativa entre o número total de lesões e o número de testes alterados de cada paciente nos grupos C-NC e A-NC, quando analisados separados e agrupados (total de 80 pacientes). Também não se evidenciou correlação do número de testes alterados de cada paciente com o número total de lesões justacorticais, profundas, à direita ou à esquerda, em ambos os grupos, quando analisados separados e agrupados (total de 80 pacientes).

Em análise de regressão linear múltipla, não foi verificada correlação entre idade, escolaridade, frequência de crises e número total de lesões simultaneamente, e o número de testes alterados de cada paciente dos grupos C-NC e do grupo A-NC, quando analisados separados e agrupados (total de 80 pacientes).

\subsection{Análise prospectiva}

Foi realizada uma reavaliação dos cinco doentes com demência encontrados no grupo A-NC. Destes cinco, quatro foram reavaliados após dois anos da abordagem inicial. Destes quatro, três pacientes ainda mantinham critérios de demência e na reavaliação por neuroimagem ainda apresentavam cistos. O único dos quatro que não mais preenchia critérios 
para demência, exibia apenas calcificações na neuroimagem controle. O quinto doente faleceu de causa distinta da patologia neurológica e não foi reavaliado.

\subsection{Poder estatístico do estudo}

Tomando os resultados do MEEM como modelo de teste de avaliação global, o cálculo do poder estatístico da comparação entre os grupos C-NC, CS e A-NC foi o seguinte: diferença entre a média mais alta (C-NC: $n=40 ; 26,85 \pm 2,90$ ) e mais baixa (A-NC: $n=40 ; 25,42 \pm 3,03$ ), assumindo-se que ambos os grupos são independentes, assim, a probabilidade de erro do tipo I, associada a esse teste na hipótese nula, foi de 0,05. O valor absoluto da diferença entre as médias dos dois grupos citados acima foi de 1,42. Levando-se em conta todos os participantes do estudo grupo (120 indivíduos no total) podemos estimar o desvio-padrão agrupado (pooled standard deviation) em um valor de 2,79, assim, sendo possível calcular o poder da comparação no estudo em um valor de 0,9998 $(99,98 \%)$. 
Tabela 3 - Frequência das alterações cognitivas encontradas nos pacientes com NC calcificada e ativa

\begin{tabular}{cccc}
\hline \multicolumn{2}{c}{ Número de pacientes (\%) } \\
\hline Demência & CCSD & Número de testes alterados \# \\
\hline C-NC & $0(0 \%)$ & $10(25,0 \%)$ & $9,4 \pm 3,1$ \\
A-NC & $5(12,5 \%)$ & $11(27,5 \%)$ & $11,6 \pm 3,9 *$ \\
\hline
\end{tabular}

LEGENDA: C-NC: neurocisticercose calcificada, A-NC: neurocisticercose ativa, CCSD : comprometimento cognitivo sem demência \# em comparação aos controles saudáveis em um total de 30 testes. * $\mathrm{p}=0.012$ 
Tabela 4 - Número total de lesões na neuroimagem de cada grupo de neurocisticercose calcificada e ativa e de seus subgrupos de acordo com as alterações cognitivas

\begin{tabular}{cccccc}
\hline & & Número médio total de lesões & & \\
& $\begin{array}{c}\text { Total } \\
(\mathbf{n}=\mathbf{4 0} \text { de cada grupo })\end{array}$ & $\begin{array}{c}\text { Demência } \\
(\mathbf{n}=\mathbf{5})\end{array}$ & $\begin{array}{c}\text { Sem demência } \\
(\mathbf{n}=\mathbf{3 5})\end{array}$ & $\begin{array}{c}\text { CCSD } \\
(\mathbf{n}=\mathbf{1 1})\end{array}$ & $\begin{array}{c}n a ̃ o \\
(\mathbf{n}=\mathbf{2 9})\end{array}$ \\
\hline C-NC & $8,80 \pm 3,20$ & - & - & $10,70 \pm 16,16$ & $8,17 \pm 12,35$ \\
A-NC & $18,73 \pm 23,35 *$ & $12,6 \pm 7,2$ & $19,6 \pm 24,7$ & - & - \\
\hline
\end{tabular}

LEGENDA: C-NC: neurocisticercose calcificada, A-NC: neurocisticercose ativa, CCSD : comprometimento cognitivo sem demência.

* $p=0.001$ (comparação total C-NC vs total A-NC); $p=0.86$ (comparação A-NC com demência vs A-NC sem demência); $p=0.36$ (comparação C-NC com CCSD vs C-NC não CCSD) 
5 DISCUSSÃO 
Esse estudo é o primeiro a realizar uma ampla avaliação cognitiva de doentes com NC calcificada, a fase mais prevalente da doença do ponto de vista epidemiológico, de forma controlada. O grupo C-NC foi pareado, quanto à idade e escolaridade a um grupo de controles saudáveis e a outro de pacientes com NC na fase cística. Os resultados mostraram que, independente da fase (calcificada ou ativa), a NC está associada a significativo declínio cognitivo. Embora a presença de demência tenha ocorrido apenas em pacientes na fase cística da doença, o comprometimento cognitivo sem demência esteve presente em ambos os grupos analisados.

Os achados obtidos em nossas amostras de doentes indicam que a NC apresenta-se com um espectro de declínio cognitivo, que varia de alteração em, pelo menos, um teste cognitivo (100\% dos casos), passando a padrão de comprometimento cognitivo amnéstico sem alteração funcional (25\% dos C-NC e 27,5\% dos A-NC), alcançando o grau de demência em $12,5 \%$ dos pacientes na fase ativa. No grupo calcificado, os domínios cognitivos mais afetados foram memória verbal, atenção e funções executivas, anormais em mais de $50 \%$ dos pacientes. O desempenho cognitivo dos doentes C-NC, embora abaixo do esperado, foi melhor que o dos doentes na fase cística. 
Ramirez-Bermudez et al. (2005) mostraram na fase longitudinal de seu estudo não controlado que a maioria dos pacientes com NC tratados com regime medicamentoso regular experimentaram uma expressiva recuperação do funcionamento cognitivo, similar ao curso de outras manifestações clínicas da doença, como crises epilépticas. Embora esses achados tenham serem bastante atraentes, neste estudo, não foi utilizado um grupo placebo. Além disso, a prescrição conjunta de corticoides influenciaria em uma análise mais profunda do real papel do albendazol no desfecho apresentado.

O grupo C-NC de nosso estudo, no momento da avaliação, apresentava-se apenas com nódulos estritamente calcificados. A RM desses doentes não mostrava lesões captantes de gadolíneo ou edema (Garcia et al., 2010). O fato realça a importância da inflamação sustentada e de outros mecanismos imunológicos, condições mais presentes durante a fase cística, em uma provável explicação do contexto clínico revelado. Sabe-se que, pela história natural da doença, lesões císticas tendem a se resolver por evolução natural na maioria dos doentes. De fato, nossos pacientes não receberam tratamento antiparasitário específico, embora esteroides e anti-histamínicos tenham sido prescritos em algum momento da evolução. Inclusive, a prescrição indiscriminada de anti-helmínticos em todos os pacientes é controversa, embora pareça levar a uma resolução radiológica mais rápida das lesões e auxilie no controle das crises epilépticas (Nash et al., 2006). Em nossa amostra, os pacientes com C-NC tiveram uma frequência de 
crises significativamente menores que os A-NC, mesmo sem uso de regime antiparasitário.

Resultados prévios publicados pelo nosso grupo mostraram que o baixo desempenho cognitivo de pacientes com NC na fase cística parecia ocorrer independente da idade, escolaridade, frequência de crises e uso de antiepilépticos (Ciampi de Andrade et al., 2010). Pacientes com NC ativa tiveram desempenho cognitivo inferior a um grupo de pacientes com epilepsia criptogênica (pareados por idade, escolaridade e com frequência de crises epilépticas semelhantes) em múltiplos domínios cognitivos. Portanto, essa diferença de desempenho deveria estar mais associada às lesões da NC em si e às suas complicações. Nossa amostra de pacientes com C-NC apresentava uma frequência de crises cerca de vinte vezes menor que o grupo epiléptico em questão, o que não nos permitiu um pareamento ideal quanto a esta variável. A ausência de comparação de nosso grupo C-NC impediu-nos da retirada de conclusões semelhantes às obtidas previamente do grupo A-NC, muito embora, o grupo C-NC apresentasse frequência crises relativamente baixas e, por conseguinte, com menor probabilidade teórica desta variável interferir nas diferenças cognitivas verificadas entre este e o controle.

Em nossa amostra, composta de 80 pacientes com NC no total, os escores cognitivos não se correlacionaram com o número de lesões ou com sua localização na neuroimagem (TC ou RM). Dois estudos prévios, também não encontraram uma correlação evidente entre os achados de neuroimagem e o desempenho cognitivo (Forlenza et al., 1997; Ciampi de 
Andrade et al., 2010). A ausência clara de correlação reforça a ideia de que o declínio cognitivo da NC, independente de sua fase, não parece ser influenciado pela carga de doença determinada só pelo número de lesões, mas, possivelmente, pela interação sinérgica entre número, localização, diferentes fases evolutivas, produção local de inflamação e edema cerebral (Góngora-Rivera et al., 2008). Esses processos parecem estar atenuados na fase calcificada, levando a uma disfunção cognitiva menos eloquente. Embora os pacientes com C-NC tivessem um número médio de lesões menores que os doentes com A-NC, esse achado deveria estar mais relacionado à evolução natural da doença, que propriamente a uma relação com o desempenho cognitivo melhor no grupo calcificado. Eventualmente, uma avaliação de uma amostra maior de pacientes permitiria confirmar a ausência da correlação entre cognição e neuroimagem de uma forma mais definitiva (Ramirez-Bermudez et al., 2005).

Uma análise de regressão linear múltipla, foi realizada, analisando uma eventual influência conjunta da idade, escolaridade, frequência de crises e número total de lesões no desempenho cognitivo de cada paciente dos grupos C-NC e A-NC, quando analisados separados e agrupados. A análise falhou ao demonstrar uma relação evidente entre estas variáveis e os resultados cognitivos dos doentes com NC. Novamente, uma amostra maior de pacientes analisados poderia eventualmente auxiliar na tentativa de aproximar variáveis clínicas e radiológicas ao fenômeno de déficit cognitivo.

O presente estudo não foi designado para investigar potenciais mecanismos implicados na disfunção cognitiva encontrada em pacientes 
com NC, tais como marcadores no LCR e citocinas. Além disso, a avaliação de pacientes virgens de tratamento antiparasitário não nos permitiu averiguar o real impacto dessa abordagem no prognóstico desses pacientes. Estudos controlados no futuro poderiam fornecer informações complementares a essas informações.

Os achados obtidos análise prospectiva dos doentes com demência poderiam indicar que a disfunção cognitiva mais acentuada da NC encontrada na fase ativa parece permanecer enquanto os cistos estão presentes. Uma replicação dessas observações em estudos subsequentes contando com um número mais extenso de pacientes com demência poderia vir a reforçar essa ideia.

Os resultados finais de nosso estudo confirmam que transtornos cognitivos são também manifestações comuns na NC calcificada. Foi demonstrado pela primeira vez de forma controlada que, independente da fase da doença, a NC leva a um espectro de anormalidades cognitivas, que variam de um mau desempenho em um único domínio, comprometimento cognitivo sem demência até ocasionalmente demência. Esses achados parecem ser mais profundos durante a fase cística da doença e tendem a se atenuar na fase calcificada. 
6 CONCLUSÕES 
1. No presente estudo, a NC em sua fase calcificada associou-se de forma significativa a comprometimento cognitivo quando comparada a controles saudáveis. Embora não tenham sido verificados pacientes com demência, observou-se uma frequência de $25 \%$ de casos com comprometimento cognitivo sem demência.

2. Os domínios cognitivos mais comprometidos nos pacientes com NC calcificada foram memória verbal, atenção e funções executivas.

3. Não foi encontrada correlação entre os achados de neuroimagem (número e localização das lesões) e a intensidade de comprometimento cognitivo nos pacientes com NC calcificada.

4. Independente de sua fase, a NC leva a um espectro de anormalidades cognitivas, que variam de um mau desempenho, em um único domínio, comprometimento cognitivo sem demência até ocasionalmente demência em sua fase ativa. Estes achados parecem ser mais profundos durante a fase cística da doença e tendem a se atenuar na fase calcificada. 
ANEXO 1 - Resultados dos testes cognitivos dos grupos C-NC, CS, A-NC

\begin{tabular}{|c|c|c|c|c|c|c|c|c|c|c|c|c|c|c|c|c|}
\hline \multicolumn{2}{|c|}{$\begin{array}{l}\text { TESTE } \\
\text { COGNTIVO }\end{array}$} & MEM & EDD & EDI & $\pi-A$ & T-Aer & $\pi-B$ & T-Ber & $\mathbf{T N}$ & BBRC- perc. & $\begin{array}{c}\text { BBRC. } \\
\text { nom }\end{array}$ & BBRC-incid. & BBRC imed.1 & $\begin{array}{l}\text { BBRC } \\
\text { imed } 2\end{array}$ & $\begin{array}{c}\text { BBRC-evoc. } \\
\text { tard }\end{array}$ & BCST-rec. \\
\hline \multirow{3}{*}{ ESCORE } & C.NC & $26,85 \pm 2,90$ & $4,67 \pm 0,92$ & $3,42+1,06$ & $67,15 \pm 28,9$ & $0,20 \pm 0,37$ & $183,38 \pm 96,2$ & $1,13 \pm 1,38$ & $13,72+1,58$ & $10,00 \pm 0,0$ & $10,00 \pm 0,00$ & $6,57 \pm 1,08$ & $9,17 \pm 0,87$ & $9,20 \pm 1,11$ & $9,00 \pm 1,26$ & $10,00 \pm 0,00^{* * x}$ \\
\hline & cs & $26,70 \pm 2,41^{*}$ & $4,77 \pm 1,00$ & $3,30 \pm 0,97$ & $58,18 \pm 24,33^{\star \star}$ & $0,37 \pm 0,71$ & $155,03 \pm 71,5^{\star \star}$ & $0,74 \pm 1,31$ & $13,10 \pm 2,14^{\star *}$ & $10,00 \pm 0,0$ & $9,98 \pm 0,16$ & $9,10 \pm 0.93$ & $9,14 \pm 0,89^{\star *}$ & $9,55 \pm 0,64$ & $9,20 \pm 0,85$ ** & $10,00 \pm 0,00^{* * x}$ \\
\hline & A-NC & $25,401+3,03^{\dagger}$ & $4,45 \pm 1,11$ & $3,05 \pm 0,88$ & $84,74 \pm 42,70^{\dagger}$ & $0,31+0,65$ & $214,78 \pm 83,02^{\dagger}$ & $1,31+1,60$ & $11,90 \pm 2,251+1958$ & $10,00 \pm 0,0$ & $9,97 \pm 0,16$ & $8,30 \pm 1,30$ & $8,71+1,01^{1+5 \S}$ & $8,92 \pm 1,27$ & $8,30 \pm 1,57+5$ & $9,80 \pm 0,61^{+1}$ \\
\hline \multicolumn{2}{|c|}{$\begin{array}{c}\text { TESTE } \\
\text { COGNTIVO }\end{array}$} & BBRC intr. & DR & Fvs & MN-imed. & $\begin{array}{c}\text { MN-evoc. } \\
\text { tar. }\end{array}$ & MN-rec. & ncs.c. & ncs-c.er. & mcs-l. & $\begin{array}{c}\text { mcs-1.- } \\
\text { er. }\end{array}$ & mcs-s.e. & nCS-e.s.er. & PC & $\begin{array}{c}\text { PCevoc } \\
\text { tard. }\end{array}$ & \\
\hline \multirow{3}{*}{ ESCORE } & C.NC & $0,02+0,16$ & $8,12+1,83$ & $16,52+4.35$ & $21,50 \pm 3,61$ & $7,10 \pm 2,07$ & $19,62+0,77^{\dagger}$ & $20,82 \pm 6,72$ & $0,02+0,16$ & $19,16 \pm 9,36$ & $0,03 \pm 0,16$ & $37,41+10,89$ & $0,49 \pm 0,85$ & $8,28 \pm 2,26$ & $6,85+2,41$ & \\
\hline & cs & $0,00 \pm 0,00$ & $7,45 \pm 2,22$ & $17,27 \pm 5,42^{* *}$ & $21,35 \pm 3,33^{\text {tht }}$ & $7,97 \pm 1,67^{\text {thk }}$ & $19,70 \pm 0,56^{\text {thk }}$ & $18,00 \pm 6,22^{\text {t* }}$ & $0,00 \pm 0,00^{\text {tx }}$ & $15,64 \pm 6,07^{*}$ & $0,07 \pm 0,35$ & $36,42+14,55^{*}$ & $0,671+1,46^{m+k}$ & $8,35 \pm 1,93$ & $6,42+2,54^{\text {*t }}$ & \\
\hline & A-NC & $0,57 \pm 2,24$ & 7,31+2,36+ & $13,72+4.29+1+53$ & $18,25 \pm 3,98^{1+1+198}$ & $6,17 \pm 2,18$ \#" & $18,52+1,831+159 s$ & $24,47 \pm 10,23^{+1}$ & $0,34 \pm 1,07$ ता & $23,06 \pm 15,86^{\dagger}$ & $0,22+0,76$ & $44,20 \pm 16,44^{+}$ & $2,11+2,63+111959$ & $7,43+2,01$ & $4,85 \pm 2,45+11989$ & \\
\hline
\end{tabular}

Resultados dos testes cognitivos expressos como média \pm desvio padrão. Neurocisticercose calcificada (C-NC), Controles Saudáveis (CS) e Neurocisiticercose ativa (A-NC). Significância estatística no teste Kruskall-Wallis entre C-NC, CS, e A-NC: ${ }^{\dagger}<0,05 ;{ }^{\dagger \dagger}<0,01 ;{ }^{\dagger \dagger}<0,001$; significância estatística no teste de Mann-Whitney entre A-NC e CS: ${ }^{*} 0,05 ;{ }^{* \star} 0,01 ;{ }^{* * *}<0,001$; significância estatística no teste de Mann-Whitney entre A-NC e C-NC: ${ }^{\S} 0,05 ;{ }^{\S \S} 0,01 ;{ }^{\S \S}<0,001$. Legendas: Mini Exame do Estado Mental (MEEM), Extensão de Dígitos Ordem Direta (EDD) e inversa (EDI), Teste de Trilhas A (TTA) e B (TTB), e seus respectivos números de erros (TT-A er e TT-B er, respectivamente), Teste de Nomeação (TN), Bateria Breve de Rastreio Cognitivo (BBRC) e seus seguintes sub-testes: percepção (perc.), nomeação (nom.), memória incidental (incid.), evocação imediata 1 and 2 (immed. 1 e 2), evocação tardia (evoc. tard.), reconhecimento (rec.) e intrusões (intr.). Teste do Desenho do Relógio (DR), Teste de Fluência Verbal Semântica (FVS), Teste de Memória Verbal (MV) e seus sub-testes:evocação imediata (imed.), evocação tardia (ecoc.tard.),e reconhecimento (rec.). Teste de Interferência de Cores de Stroop (TICS) e seus sub-testes: cores (c.), leitura (I.),e efeito Stroop (e.s.), seguidos de respectivos número de erros (er.). Praxia Construtiva (PC) e sua evocação tardia (evoc.tard.). 
ANEXO 2 - Resultados dos testes cognitivos e funcionais de indivíduos do grupo controle saudável (CS) e o número de pacientes do grupo C-NC com escores alterados

\begin{tabular}{|c|c|c|c|c|c|c|c|c|c|c|c|c|c|c|c|}
\hline TESTE COGNITVO & MEM & EDD & 由I & TT-A & TT-Aer & $\pi-B$ & T-Ber & $\mathbf{T N}$ & BBRC- perc. & BBRC- nom & BBRC-incid. & $\begin{array}{l}\text { BBRC } \\
\text { imed.1 }\end{array}$ & $\begin{array}{l}\text { BBRC-imed. } \\
2\end{array}$ & $\begin{array}{l}\text { BBRC-evoc. } \\
\text { tard }\end{array}$ & BCST-rec. \\
\hline $\begin{array}{l}\text { Escore médio } \\
\text { cS } \pm \text { d.p.p. } \\
\text { (extensão); IC }\end{array}$ & $\begin{array}{l}26,70 \pm 2,41 \\
(22-30) ; 0,75\end{array}$ & $\begin{array}{l}4,77 \pm 1,00 \\
(3-7) ; 0,31\end{array}$ & $\begin{array}{l}3,30 \pm 0,97 \\
(2-6) ; 0,30\end{array}$ & $\begin{array}{l}58,18+24,33 \\
(22-135) ; 7,64\end{array}$ & $\begin{array}{l}0,37 \pm 0,71 \\
(0-3) ; 0,22\end{array}$ & $\begin{array}{l}155,03 \pm 71,5 \\
\text { (55-300); } 22,52\end{array}$ & $\begin{array}{l}0,74 \pm 1,31 \\
(0-6) ; 0,41\end{array}$ & $\begin{array}{l}13,10+2,14 \\
(5-15) ; 0,67\end{array}$ & $10 \pm 0,0$ & $\begin{array}{l}9,97 \pm 0,16 \\
(9-10) ; 0,05\end{array}$ & $\begin{array}{l}6,85+1,27 \\
(4-10) ; 0,39\end{array}$ & $\begin{array}{l}9,10 \pm 0,93 \\
(7-10) ; 0,29\end{array}$ & $\begin{array}{l}9,55+0,64 \\
(8-10) ; 0,20\end{array}$ & $\begin{array}{l}9,20 \pm 0,85 \\
(7-10) ; 0,26\end{array}$ & $10 \pm 0,0$ \\
\hline $\begin{array}{l}\text { Número de } \\
\text { pacientes comc } \\
\text { NC com scores } \\
\text { alterados (\%) }\end{array}$ & $9(22,5 \%)$ & $20(50 \%)$ & $27(67,5 \%)$ & $13(32,5 \%)$ & $32(80 \%)$ & $15(37,5 \%)$ & $23(57,5 \%)$ & $10(20 \%)$ & 0 & 0 & $20(50 \%)$ & $8(20 \%)$ & $17(42,5 \%)$ & $11(27,5 \%)$ & 0 \\
\hline TESTE COGNITIVO & BBRC intr. & DR & Fvs & MN-imed. & MN-evoc. tar. & MN-rec. & ncs.c. & ncs-c.er. & ncs-l. & nCS-1.er. & ncs-s.e. & $\begin{array}{l}\text { ncs-es.- } \\
\text { er. }\end{array}$ & PC & $\begin{array}{l}\text { PCevoc } \\
\text { tard. }\end{array}$ & QAPP \\
\hline $\begin{array}{l}\text { Escore médio } \\
\text { CS } \pm \text { d.p. } \\
\text { (extensão); IC }\end{array}$ & $0,0 \pm 0,0$ & $\begin{array}{l}7,45+2,22 \\
(2-10) ; 0,69\end{array}$ & $\begin{array}{l}17,27 \pm 5,42 \\
(8-31) ; 1,68\end{array}$ & $\begin{array}{l}21,35 \pm 3,33 \\
(13-27) ; 1,03\end{array}$ & $\begin{array}{l}7,97 \pm 1,67 \\
(4-10) ; 0,51\end{array}$ & $\begin{array}{l}19,70 \pm 0,56 \\
(18-20) ; 0,17\end{array}$ & $\begin{array}{c}18,00 \pm 6,22 \\
(11-35) ; 1,93\end{array}$ & $0,0 \pm 0,0$ & $\begin{array}{l}15,64 \pm 6,07 \\
(9-39) ; 1,91\end{array}$ & $\begin{array}{l}0,07 \pm 0,35 \\
(0-2) ; 0,11\end{array}$ & $\begin{array}{l}36,42+14,55 \\
(18-76) ; 4,51\end{array}$ & $\begin{array}{l}0,67 \pm 1,46 \\
(0-6) ; 0,45\end{array}$ & $\begin{array}{l}8,35+1,93 \\
(5-11) ; 0,60\end{array}$ & $\begin{array}{l}6,42+2,54 \\
(1-10) ; 0,79\end{array}$ & $10,0 \pm 0,0$ \\
\hline $\begin{array}{l}\text { Número de } \\
\text { pacientes comc } \\
\text { NC com scores } \\
\text { alterados (\%) }\end{array}$ & 0 & $6(15 \%)$ & $16(40 \%)$ & $16(40 \%)$ & $21(52,5 \%)$ & $10(20 \%)$ & $12(30 \%)$ & 0 & $10(25 \%)$ & 0 & $15(37,5 \%)$ & $26(65 \%)$ & $16(40 \%)$ & $11(27,5 \%)$ & 0 \\
\hline
\end{tabular}

Legenda. Controles Saudáveis (CS), Neurocisticercose calcificada (C-NC), desvio padrão (d.p.), intervalo de confiança (IC). Mini Exame do Estado Mental (MEEM), Extensão de Dígitos Ordem Direta (EDD) e inversa (EDI), Teste de Trilhas A (TTA) e B (TTB), e seus respectivos números de erros (TT-A er e TT-B er, respectivamente), Teste de Nomeação (TN), Bateria Breve de Rastreio Cognitivo (BBRC) e seus seguintes sub-testes: percepção (perc.), nomeação (nom. incidental (incid.), evocação imediata 1 and 2 (immed. 1 e 2), evocação tardia (evoc. tard.), reconhecimento (rec.) e intrusões (intr.). Teste do Desenho do Relógio (DR), Teste de Fluência Verbal Semântica (FVS), Teste de Memória Verbal (MV) e seus sub-testes:evocação imediata (imed.), evocação tardia (ecoc.tard.),e reconhecimento (rec.). Teste de Interferência de Cores de Stroop (TICS) e seus sub-testes: cores (c.), leitura (I.), e efeito Stroop (e.s.), seguidos de respectivos número de erros (er.). Praxia Construtiva (PC) e sua evocação tardia (evoc.tard.) e Questionário de Atividades Funcionais de Pfeffer (QAFP). 
ANEXO 3 - Resultados dos testes cognitivos e funcionais de indivíduos do grupo controle saudável (CS) e o número de pacientes do grupo NC ativa (grupo A-NC) com escores alterados

\begin{tabular}{|c|c|c|c|c|c|c|c|c|c|c|c|c|c|c|c|}
\hline TESTE COGNTIVO & MEM & EDD & EDI & $\pi-A$ & TT-Aer & $\pi-B$ & T-Ber & $\mathbf{T N}$ & BBRC perc. & BBRC- nom & BBRC-incid. & $\begin{array}{l}\text { BBRC } \\
\text { imed.1 }\end{array}$ & $\begin{array}{l}\text { BBRC-imed. } \\
2\end{array}$ & $\begin{array}{l}\text { BBRC-evoc. } \\
\text { tard }\end{array}$ & BCST-rec. \\
\hline $\begin{array}{l}\text { Escore médio } \\
\text { CS士d.p. } \\
\text { (extensão); ic }\end{array}$ & $\begin{array}{l}26,70 \pm 2,41 \\
(22-30) ; 0,75\end{array}$ & $\begin{array}{l}4,77 \pm 1,00 \\
(3-7) ; 0,31\end{array}$ & $\begin{array}{l}3,30 \pm 0,97 \\
(2-6) ; 0,30\end{array}$ & $\begin{array}{c}58,18 \pm 24,33 \\
(22-135) ; 7,64\end{array}$ & $\begin{array}{l}0,37 \pm 0,71 \\
(0-3) ; 0,22\end{array}$ & $\begin{array}{l}155,03+71,5 \\
(55-300) ; 22,52\end{array}$ & $\begin{array}{l}0,74 \pm 1,31 \\
(0-6) ; 0,41\end{array}$ & $\begin{array}{l}13,10 \pm 2,14 \\
(5-15) ; 0,67\end{array}$ & $10 \pm 0,0$ & $\begin{array}{l}9,97 \pm 0,16 \\
(9-10) ; 0,05\end{array}$ & $\begin{array}{l}6,85+1,27 \\
(4-10) ; 0,39\end{array}$ & $\begin{array}{l}9,10 \pm 0,93 \\
(7-10) ; 0,29\end{array}$ & $\begin{array}{l}9,55 \pm 0,64 \\
(8-10) ; 0,20\end{array}$ & $\begin{array}{l}9,20 \pm 0,85 \\
(7-10) ; 0,26\end{array}$ & $10 \pm 0,0$ \\
\hline $\begin{array}{l}\text { Número de } \\
\text { pacientes com NC } \\
\text { A com scores } \\
\text { alterados }(\%)\end{array}$ & $19(47,5 \%)$ & $21(52,5 \%)$ & $32(80 \%)$ & $8(20 \%)$ & $31(77,5 \%)$ & $9(22,5 \%)$ & $15(37,5 \%)$ & $23(57,5 \%)$ & 0 & $1(2,5 \%)$ & $19(47,5 \%)$ & 19(47,5\%) & $23(57,5 \%)$ & $17(42,5 \%)$ & 0 \\
\hline TESTE COGNTIVO & BBRC intr. & $\mathbf{T R}$ & Fvs & MN-imed. & MN-evoc. tar. & MN-rec. & mcs.c. & ncs-c-er. & ncs-l. & nCS-1.er. & ncS-s.e. & $\begin{array}{l}\text { mos-es.- } \\
\text { er. }\end{array}$ & PC & $\begin{array}{l}\text { PCevoc } \\
\text { tard. }\end{array}$ & PFAQ \\
\hline $\begin{array}{l}\text { Escore médio } \\
\text { CS } \pm \text { d.p. } \\
\text { (extensão); IC }\end{array}$ & $0,0 \pm 0,0$ & $\begin{array}{l}7,45+2,22 \\
(2-10) ; 0,69\end{array}$ & $\begin{array}{l}17,27 \pm 5,42 \\
(8-31) ; 1,68\end{array}$ & $\begin{array}{c}21,35 \pm 3,33 \\
(13-27) ; 1,03\end{array}$ & $\begin{array}{l}7,97 \pm 1,67 \\
(4-10) ; 0,51\end{array}$ & $\begin{array}{l}19,70 \pm 0,56 \\
(18-20) ; 0,17\end{array}$ & $\begin{array}{c}18,00 \pm 6,22 \\
(11-35) ; 1,93\end{array}$ & $0,0 \pm 0,0$ & $\begin{array}{l}15,64 \pm 6,07 \\
(9-39) ; 1,91\end{array}$ & $\begin{array}{l}0,07 \pm 0,35 \\
(0-2) ; 0,11\end{array}$ & $\begin{array}{l}36,42+14,55 \\
(18-76) ; 4,51\end{array}$ & $\begin{array}{l}0,67 \pm 1,46 \\
(0-6) ; 0,45\end{array}$ & $\begin{array}{l}8,35+1,93 \\
(5-11) ; 0,60\end{array}$ & $\begin{array}{c}6,42+2,54 \\
(1-10) ; 0,79\end{array}$ & $10,0 \pm 0,0$ \\
\hline $\begin{array}{l}\text { Número de } \\
\text { pacientes com NC } \\
\text { A com scores } \\
\text { alterados ( } \% \text { ) }\end{array}$ & 0 & $13(32,5 \%)$ & $30(75 \%)$ & $27(67,5 \%)$ & $28(70 \%)$ & $23(57,5 \%)$ & $9(22,5 \%)$ & 0 & $10(25 \%)$ & 0 & $9(22,5 \%)$ & $13(32,5 \%)$ & $16(40 \%)$ & $23(57,5 \%)$ & $5(12,5 \%)$ \\
\hline
\end{tabular}

Legenda. Controles Saudáveis (CS), Neurocisticercose ativa (A), desvio padrão (d.p.), intervalo de confiança (IC). Mini Exame do Estado Mental (MEEM), Extensão de Dígitos Ordem Direta (EDD) e inversa (EDI), Teste de Trilhas A (TTA) e B (TTB), e seus respectivos números de erros (TT-A er e TT-B er, respectivamente), Teste de Nomeação (TN), Bateria Breve de Rastreio Cognitivo (BBRC) e seus seguintes sub-testes: percepção (perc.), nomeação (nom.), memória incidental (incid.), evocação imediata 1 and 2 (immed. 1 e 2), evocação tardia (evoc. tard.), reconhecimento (rec.) e intrusões (intr.). Teste do Desenho do Relógio (DR), Teste de Fluência Verbal Semântica (FVS), Teste de Memória Verbal (MV) e seus sub-testes:evocação imediata (imed.), evocação tardia (ecoc.tard.), e reconhecimento (rec.). Teste de Interferência de Cores de Stroop (TICS) e seus sub-testes: cores (c.), leitura (I.),e efeito Stroop (e.s.), seguidos de respectivos número de erros (er.). Praxia Construtiva (PC) e sua evocação tardia (evoc.tard.) e Questionário de Atividades Funcionais de Pfeffer (QAFP). 
8 REFERÊNCIAS 
Abraham R. Neurocisticercose: relação entre dosagem de antígenos de Taenia no líquido cefalorraquidiano e imagem através de ressonância magnética. [Tese Doutorado]. São Paulo: Faculdade de Medicina, Universidade de São Paulo, 2006.

Agapejev S. Aspectos clínicos-epidemiológicos da neurocisticercose no Brasil. Arq Neuropsiquiatr . 2003; 61:822-28.

Alvarez-Rodríguez E, Torres-Gárate R, Cabello J, Lozano Tonkin C . Neurocysticercosis in Spain. Rev Clin Esp. 2005; 205 :518.

Boppré MC, Wille PR, Guarnieri R, et al. Cognitive performance of patients with epilepsy and calcified neurocysticercotic lesions: a case-control study. Epilepsy Behav. 2001; 2:558-562.

Bertolucci PHF, Brucki SM, Campacci SR, Juliano Y. The Mini-Mental State Examination in a general population: impact of educational status. Arq Neuropsiquiatr. 1994;52:1-7.

Bertolucci PHF, Okamoto HI, Toniolo Neto J, Ramos LR, Brucki SM. Rev Psiq Clin. 1998; 25:80-3.

Bragazza LM, Vaz AJ, Passos AD, et al. Frequency of serum anti-cysticercus antibodies in the population of a rural Brazilian community (Cássia dos coqueiros, SP) determined by Elisa and immunoblotting using Taenia crassiceps antigens. Rev Inst Med Trop Sao Paulo. 2002;44:7-12.

Brucki, SM, Nitrini R, Caramelli P, Bertolucci PH, Okamoto IH. Suggestions for utilization of the mini-mental state examination in Brazil. Arq Neuropsiquiatr. 2003; 61: 777-81. 
Canelas HM . Neurocisticercose: incidência, diagnóstico e formas clínicas. Arq Neuropsiquiatr. 1962; 20: 1-16.

Carpio A. Neurocysticercosis: an update. The Lancet Infectious Diseases. 2002; 2: 751-62.

Ciampi de Andrade D, Rodrigues CL, Abraham R, Castro LHM, Livramento JA, Machado LR, Leite CC, Caramelli P. Cognitive impairment and dementia in neurocysticercosis: a cross-sectional controlled study. Neurology. 2010;74:1288-95.

Cruz ME, Schantz PM, Cruz I, Espinosa P, Preux PM, Cruz A, Benitez W, Tsang VC, Fermoso J, Dumas M. Epilepsy and neurocysticercosis in an Andean community. Int J Epidemiol. 1999; 28:799-803.

Del Brutto $\mathrm{OH}$, Rajshekhar V, White AC Jr, et al. Proposed diagnostic criteria for neurocysticercosis. Neurology. 2001; 57:177-83.

Del la Garza Y, Graviss EA, Daver NG, Gambarin KJ, Shandera WX, Schantz PM, White AC Jr Epidemiology of neurocysticercosis in Houston, Texas. Am J Trop Med Hyg. 2005;73:766-70.

Dixon HBF, Hargreaves WH. Cysticercosis (Taenia solium). A further ten years' clinical study, covering 284 cases. Quarterly Journal of Medicine. 1945; 13:107-21.

Dixon HBF, Lipscomb FM. Cysticercosis: an analyses and follow-up of 450 cases. Medical Research Council Special Report Series. 1961; 288:1-58.

Ewing JA. Detecting alcoholism. The CAGE questionnaire. JAMA. 1984; 252:1905-7. 
Folstein MF, Folstein SE, McHugh PR. "Mini-mental state". A practical method for grading the cognitive state of patients for the clinician. J Psychiatr Res. 1975; 12: 189-98.

Forlenza OV, Vieira AHG, Nóbrega JPS, et al. Psychiatric manifestations in neurocysticercosis: a study of 38 patients from a neurology clinic in Brazil. $J$ Neurol Neurosurg Psychiatry . 1997;62:612- 16.

Garcia HH, Gonzalez AE, Gilman RH, for The Cysticercosis Working Group in Peru. Diagnosis, treatment and control of Taenia solium cysticercosis. Curr Opin Inf Dis. 2003;16: 411-19.

Garcia HH, Pretell EJ, Gilman RH, Martinez SM, Moulton LH, Del Brutto OH, Herrera G, Evans CA, Gonzalez AE; Cysticercosis Working Group in Peru. A trial of antiparasitic treatment to reduce the rate of seizures due to cerebral cysticercosis. N Engl J Med. 2004; 350:249-58.

Garcia HH, Del Brutto OH. Cysticercosis Working Group in Peru. Neurocysticercosis: updated concepts about an old disease. Lancet Neurol. 2005; 4: 653-61.

García HH, Gonzalez AE, Rodriguez S, Tsang VC, Pretell EJ, Gonzales I, Gilman RH; Cysticercosis Working Group in Peru. Neurocysticercosis: unraveling the nature of the single cysticercal granuloma. Neurology. 2010; 75:654-8.

Garcia-Noval J, Moreno E, de Mata F, et al. An epidemiological study of epilepsy and epileptic seizures in two rural Guatemalan communities. Ann Trop Med Parasitol. 2001; 95:167-75.

Góngora-Rivera F, Soto-Hernández JL, Guevara P, Sotelo-Morales J In neurocysticercosis, CSF cytokines correlate with cerebral blood flow velocities.. Neurology. 2008;71:1119-22. 
Griesinger W. Fortgesetzte Beobachtung über Hirnkrankheiten, Cysticerken und ihre Diagnose. Arch Kinderheilk. 1862;3:207-40.

Kraft R. Cysticercosis: an emerging parasitic disease. Am Fam Physician. 2007; 76:91-6.

Lucato LT, Guedes MS, Sato JR, Bacheschi LA, Machado LR, Leite CC. The role of conventional MR imaging sequences in the evaluation of neurocysticercosis: impact on characterization of the scolex and lesion burden. ANJR Am J Neuroradiol. 2007;28:1501-4.

Más-Sesé G, Vives-Piñera I, Fernández-Barreiro A, et al. C. A descriptive study of eurocysticercosis in a tertiary care hospital. Rev Neurol. 2008;46:194-6.

Medina MT, Durón RM, Martínez L, et al .Prevalence, incidence, and etiology of epilepsies in rural Honduras: the Salamá Study. Epilepsia. 2005; 46:12431.

Montano SM, Villaran MV, Ylquimiche L, et al . Cysticercosis Working Group in Peru. Neurocysticercosis: association between seizures, serology, and brain CT in rural Peru. Neurology. 2005;65:229-33.

Morris JC, Heyman A, Mohs RC, et al. The Consortium to Establish a Registry for Alzheimer's Disease (CERAD). Part 1. Clinical and neuropsychological assessment of Alzheimer's disease. Neurology. 1989; 39: $1159-65$.

Nash TE, Del Brutto OH, Butman JA, et al. Calcific neurocysticercosis and epileptogenesis. Neurology. 2004;62:1934-8.

Nitrini R, Caramelli P, Bottino CM, Damasceno BP, Brucki SM, Anghinah R; Academia Brasileira de Neurologia. Diagnosis of Alzheimer's disease in 
Brazil: cognitive and functional evaluation. Recommendations of the Scientific Department of Cognitive Neurology and Aging of the Brazilian Academy of Neurology. Arq Neuropsiquiatr. 2005; 63:720-7.

Nitrini R, Caramelli P, Bottino CM, Damasceno BP, Brucki SM, Anghinah R; Academia Brasileira de Neurologia. Diagnosis of Alzheimer's disease in Brazil: diagnostic criteria and auxiliary tests. Recommendations of the Scientific Department of Cognitive Neurology and Aging of the Brazilian Academy of Neurology. Arq Neuropsiquiatr. 2005; 63:713-9.

Nitrini R, Caramelli P, Herrera $\mathrm{E} \mathrm{Jr}$, et al. Incidence of dementia in a community-dwelling Brazilian population. Alzheimer Dis Assoc Disord. 2004;18:241-6.

Nitrini R, Lefèvre BH, Mathias SC, et al. Neuropsychological tests of simple application for diagnosing dementia. Arq Neuropsiquiatr. 1994; 52:457-65.

Ong S, Talan DA, Moran GJ, et al. Neurocysticercosis in radiographically imaged seizure patients in U.S. emergency departments. Emerg Infect Dis. 2002;8:608-13.

Poeschl P, Janzen A, Schuierer G, Winkler J, Bogdahn U, Steinbrecher A. Calcified neurocysticercosis lesions trigger symptomatic inflammation during antiparasitic therapy. AJNR Am J Neuroradiol. 2006;27:653-5.

Pfeffer RI, Kurosaki TT, Harrah CH, Chance JM, Filis S. Measurement of functional activities in older adults in the community. J Gerontol, 1982; 37: 323-29.

Ramirez-Bermudez J, Higuera J, Sosa AL, Lopez-Meza E, Lopez-Gomez M, Corona T. Is dementia reversible in patients with neurocysticercosis? $J$ Neurol Neurosurg Psychiatry. 2005; 76: 1164-66. 
Román G, Sotelo J, Del Brutto $\mathrm{O}$, et al. A proposal to declare neurocysticercosis an international reportable disease. Bull World Health Organ. 2000; 78: 399-406

Ruiz S, García-Vázquez E, Picazo R, Hernández A, Herrero JA, Gómez J. Neurocysticercosis in Murcia (Spain). Rev Clin Esp. 2011; 211:133-38.

Sandyk R, Bamfor C, lacono RP, Gillman MA. Cerebral cysticercosis presenting as progressive dementia. International Journal of Neuroscience. 1987; 35: 251-54.

Saunders JB, Aasland OG, Babor TF, de la Fuente JR, Grant M. Development of the Alcohol Use Disorders Identification Test (AUDIT): WHO Collaborative Project on Early Detection of Persons with Harmful Alcohol Consumption--II. Addiction. 1993; 88:791-804.

Serpa JA, Graviss EA, Kass JS, White AC Jr. Neurocysticercosis in Houston, Texas: an update. Medicine (Baltimore). 2011;90:81-6.

Shandera WX, White AC Jr, Chen JC, Diaz P, Armstrong R. Neurocysticercosis in Houston, Texas. A report of 112 cases. Medicine (Baltimore). 1994; 73:37-52.

Sorvillo F, Wilkins P, Shafir S, Eberhard M. Public health implications of cysticercosis acquired in the United States. Emerg Infect Dis. 2011; 17:1-6.

Sotelo J. Neurocysticercosis-is the elimination of parasites beneficial? N Engl J Med. 2004;350:280-2.

Sotelo J, Del Brutto OH, Roman GC. Cysticercosis. Curr Clin Top Infect Dis. 1996; 16:240-59. 
Sotelo J, Guerrero V, Rubio F. Neurocysticercosis: a new classification based on active and inactive forms. Arch Intern Med. 1985; 145:442-5.

Spina-França A, Livramento JA, Machado LR. Cysticercosis of the central nervous system and cerebrospinal fluid. Immunodiagnosis of 1573 patients in 63 years (1929-1992). Arq Neuropsiquiatr .1993;51: 16-20.

Stroop JR. Studies of interference in serial verbal reactions. Journal of Experimental Psychology. 18, 643-62.

Takanayagui OM. Clinical aspects of neurocysticercosis: analysis of 500 cases. Arq Neuropsiquiatr. 1983;41:50-63.

Takayanagui OM. Neurocysticercosis. I. Clinical and laboratory course of 151 cases. Arq Neuropsiquiatr. 1990; 48:1-10.

Takayanagui OM, Leite JP. Neurocysticercosis. Rev Soc Bras Med Trop. 2001; 34:283-90.

Tavares Jr AR. Psychiatric disorders in neurocysticercosis. $\mathrm{Br} \mathrm{J}$ Psychiatry. 1993; 163:839.

Terra-Bustamante VC, Coimbra ER, Rezek KO, et al. Cognitive performance of patients with mesial temporal lobe epilepsy and incidental calcified neurocysticercosis. J Neurol Neurosurg Psychiatry. 2005; 76:1080-3.

Tretiakoff C, Pacheco e Silva AC. Contribuicão para o estudo da cysticercose cerebral e em particular das lesões cerebrais tóxicas à distância n’esta afeccão. Memórias do Hospício de Juqueri. 1924;1:37- 66.

Trzepacz PT, Baker RW, Greenhouse J. A symptom rating scale for delirium. Psychiatry Res. 1988;23:89-97. 
Townes JM, Hoffmann CJ, Kohn MA. Neurocysticercosis in Oregon, 19952000. Emerg Infect Dis. 2004;10:508-10.

Varma A, Gaur KJ. The clinical spectrum of neurocysticercosis in the Uttaranchal region. J Assoc Physicians India. 2002; 50:1398-400.

Vaz AJ. Neurocisticercose: Aspectos Biológicos, Imunológicos e Epidemiológicos. In Machado LR, Livramento JA, Spina-França A, Nóbrega JPS (eds). Neuroinfecção 96. São Paulo: Clínica Neurológica HC/FMUSP, 1996: 181-192.

Wechsler D. ed. Wechsler Memory Scale - Revised. Psychological Corporation: San Antonio, Texas, Estados Unidos da América, 1981. 